\title{
Eine Bestandesaufnahme der postkolonialen Schweiz ${ }^{1}$
}

\author{
Patricia Purtschert, Barbara Lüthi und Francesca Falk
}

Die weit verbreitete Meinung, wonach die Schweiz mit dem Kolonialismus nichts zu tun gehabt habe, schlägt sich im helvetischen Kontext nicht nur im Geschichtsunterricht nieder, sondern auch in der medialen Berichterstattung, in der Außenpolitik oder in alltäglichen Gesprächen. Selbst in der neueren Historiographie fehlt eine Reflexion der Schweizer Position in der kolonialen Konstellation fast gänzlich. ${ }^{2}$ Ist es im Kontrast zu diesem weitgehend fehlenden Problembewusstsein angebracht, so fragten wir Herausgeberinnen uns, von einer »postkolonialen Schweiz«zu sprechen? Und wenn ja, wie lässt sich dieser Begriff begründen? Denn auch wenn man, wie wir es tun, davon ausgeht, dass Schweizer Akteure und Akteurinnen auf vielfältige Weise in die kolonialen Geschehnisse verstrickt waren, stellt sich die Frage: Ergibt es Sinn, postkoloniale Ansätze auf ein Land anzuwenden, das als Nationalstaat nie formale Kolonien besessen hat?

Pointiert herausstellen lässt sich die Schwierigkeit, über die Schweiz als kolonialen und postkolonialen Raum nachzudenken, im Vergleich mit einer benachbarten ehemaligen Kolonialmacht. Im französischen Kontext, in dem die Rezeption postkolonialer Ansätze ebenfalls erst vor wenigen Jahren eingesetzt hat, können koloniale Praktiken und ihre postkolonialen Ausläufer direkt an die eigene Kolonialgeschichte zurückgebunden werden. So wird die breite Ausstellungspraxis

1 Die Herausgeberinnen bedanken sich bei Harald Fischer-Tiné, Eva Keller, Gesine Krüger, Marina Lienhard, Martin Mühlheim, Jovita dos Santos Pinto, Jana Tschurenev und Yves Winter für die hilfreichen Kommentare. Marina Lienhard und Jovita dos Santos Pinto danken wir zudem für die Recherchearbeiten. Wertvolle Rückmeldungen gaben uns zudem die Teilnehmerinnen und Teilnehmer der Übung "Postkoloniale Perspektiven in der politischen Theorie", die am Historischen Seminar in Basel im Frühlingssemester 2011 stattfand.

2 | So kommen weder Maissen noch Reinhard auf den Kolonialismus zu sprechen, vgl. Maissen, Geschichte der Schweiz; Reinhardt, Geschichte der Schweiz. Ein kurzer Hinweis auf den "verdeckten Kolonialismus" der Schweiz findet sich in Comité pour une Nouvelle Histoire de la Suisse, Geschichte der Schweizer, S. 712. 
kolonialer Gegenstände im 19. und frühen 20. Jahrhundert mit der Notwendigkeit in Verbindung gebracht, die Bevölkerung für das koloniale Unterfangen zu gewinnen: »le lobby colonial a travaillé sans relâche pour convaincre un public peu enthousiaste des mérites de l'expansion outre-mer « ${ }^{3}$. Weiter wird darüber geforscht, »comment la colonie >fait retour en métropole « ${ }^{4}$. Und es wird gefragt, wie die im kolonialen Setting gemachten Erfahrungen die Vorstellungen von Anderen, »les imaginaires et les conceptions sur l'Autre «", in Frankreich geprägt haben. Die Vorannahmen, die in diesen Fragestellungen zum Ausdruck kommen, scheinen im französischen Kontext durchaus sinnvoll zu sein: Es geht darum zu verstehen, wie sich die Kolonialpolitik in der eigenen Bevölkerung durchsetzen ließ, welche Rückwirkungen der Kolonialismus auf Frankreich hatte und wie die Wahrnehmung von Anderen und vom Fremden maßgeblich vom Kolonialismus geprägt wurde. Auch die Frage, was für Widerstände gegenüber einer nationalen Kolonialpolitik in Frankreich selbst auszumachen sind, beispielsweise von Seiten der Kommunistinnen, Existentialisten und Surrealistinnen, liegt auf der Hand. ${ }^{6}$

Wie aber lassen sich die Ausformungen und Effekte der kolonialen Regimes in einem Land wie der Schweiz fassen? Wie lassen sich die beliebten Ausstellungen kolonialer Gegenstände um 1900 in der Schweiz deuten, die enorme Sammlertätigkeit von Schweizer Forschenden, die Völkerschauen7, die exotischen Postkarten, die Reiseliteratur? Wie lassen sich Auswirkungen und Rückwirkungen der kolonialen Konstellation auf die Schweiz bestimmen? Besteht auch in der Schweiz so etwas wie ein »koloniales Imaginäres«, das die Wahrnehmung von Anderen bis hinein in unsere Gegenwart strukturiert? Und wenn ja, wie müssen diese Aspekte für ein Land gedacht werden, das sich selbst bis heute weitgehend außerhalb der kolonialen Ereignisse wähnt?

Auch in der Schweiz gab es Ende des 19. Jahrhunderts Stimmen, die eine Beteiligung des Bundes an konkreten Kolonisierungsprojekten forderten. So schlug 1884, also in der Hochphase des Scramble for Africa, der Nationalrat Friedrich Salomon Vögelin ${ }^{8}$ im Rahmen eines Vorstoßes für die Revision der Bundesverfassung vor, die Schweizerinnen und Schweizer in den Kolonien unter einen direkteren Schutz der Eidgenossenschaft zu stellen.

3 | Aldrich, "Musée Colonial Impossible", S. 89.

4 | Bancel/Blanchard, "Avant-Propos", S. 13.

5 | Ebd., S. 12.

6 | Siehe dazu die Ausstellung Exotiques expositions. Les expositions universelles et les cultures extra-européennnes, France 1855-1937, die im Frühling und Sommer 2010 in den Archives nationales in Paris stattfand. Siehe auch den Katalog zur Ausstellung: Demeulenaere-Douyère, Exotiques expositions.

7 | Siehe dazu den Artikel von Christof Dejung in diesem Band.

8 | Betulius, Vögelin. 
"Unaufhaltsam drängt das öffentliche Bewusstsein dahin, dass der Bund, wie er die Auswanderungs-Agenturen überwacht, ja auch den Auswanderern selbst seine Obsorge und seine Hülfe angedeihen lasse und dass er sich auch bei der Errichtung von Kolonien mit seiner Autorität, seiner Vermittlung und, wenn nöthig, seiner Unterstützung beteilige. Diejenigen, welche den Boden der Heimat für immer zu verlassen den schweren Entschluss gefasst haben, hören damit nicht auf, Kinder unseres Vaterlandes zu sein und Anspruch auf seinen Schutz und seine Obsorge zu haben. Dieses Gefühl muss im Schweizervolke durchschlagen und seinen feierlichen und verbindlichen Ausdruck in der Verfassung finden. " ${ }^{9}$

Vögelin forderte deshalb eine »direkte legislatorische und materielle Betheiligung des Bundes bei Auswanderungs- und Kolonialwesen ${ }^{10}$, beispielsweise indem das Fabrikgesetz entsprechend erweitert werde. Bundesrat Numa Droz, Vorsteher des Handels- und Landwirtschaftsdepartements, stand einer solchen Beteiligung skeptisch gegenüber und entgegnete: »Mit der Beteiligung an Kolonisationsunternehmungen würde der Bund eine Verantwortung übernehmen, der er unter Umständen absolut nicht gerecht werden könnte. Darüber sind alle Nationalökonomen einig, dass, um zu kolonisiren, ein Stat ein Küstenland sein und also auch eine Flotte haben muss. «"11 Vögelins Vorstoß ist vorsichtig zu interpretieren; es wäre wohl falsch, den Zürcher Professor für Kulturgeschichte und Initiator des Landesmuseums, der »sein Herz den untern Volksschichten « ${ }^{12}$ schenkte, vorschnell zu einer der treibenden Kräfte der Schweizer Kolonisierung zu stilisieren. Dennoch wäre es interessant, die jeweils gewählten Begrifflichkeiten auf ihre Implikationen hin zu untersuchen: Wer spricht von Auswanderung, wer wann und unter welchen Umständen von Kolonisierung? Vögelins Dokumente oder auch die Zeitschrift Der Kolonist ${ }^{13}$ einer präzise argumentierenden und kontextualisierenden Diskursanalyse zu unterziehen, wäre ein vielversprechendes Forschungsvorhaben; gerade der spezifische Sprachgebrauch könnte interessante Rückschlüsse auf das Verhältnis von Schweizer Akteuren und Akteurinnen zum Kolonialismus ermöglichen.

9 | Vögelin, "Rede", S. 59. Wir folgen jeweils der überlieferten Schreibweise und verzichten aus Gründen der Lesbarkeit auf das Wort "sic".

10 | Ebd., S. 60.

11 | Droz, "Revision ", S. 619.

12 | Feller/Bonjour, Geschichtsschreibung, S. 723.

13 | Beatrice Ziegler zählt drei Namensvarianten der Zeitschrift auf: Vom 06.06.1851 bis 1853 lief sie unter dem Namen Der Colonist, Organ zum Schutze, Beistand und Belehrung schweizerischer Auswanderer, vom 01.01.1854 bis Neujahr 1855 unter der Bezeichnung Der Kolonist, Organ für die schweizerische Auswanderung und vom 01.01.1855 bis Ende des dritten Quartals 1857 war schließlich die Benennung Der Kolonist, Organ für die schweizerische Auswanderung, insbesondere nach Nord- und Südamerika gebräuchlich. Siehe dazu Ziegler, Schweizer statt Sklaven, S. 458. 


\section{Postcolonial Studies: Ein anderer Blick auf die Moderne}

In den letzten Jahren hat sich eine wachsende Zahl von wirtschaftshistorischen Studien mit der Beteiligung von Schweizer Akteuren am transatlantischen Sklaven- und Kolonialhandel beschäftigt. ${ }^{14}$ Diese Studien eröffnen nicht nur ein neues Diskussionsfeld, sie nehmen auch bestehende Forschungen auf und verleihen ihnen neue Relevanz: Ohne militärische Verantwortung tragen zu müssen, profitiere die Schweiz von kolonialen Konstellationen, meinte Richard Fritz Behrendt bereits in den $1930 e r$ Jahren. ${ }^{15}$ Die Schweiz wurde dabei, auch wegen ihrer begrenzten territorialen Ausdehnung und geopolitischen Bedeutung, von den formalen Kolonialmächten nicht als Konkurrenz wahrgenommen: »es ist selbstverständlich, dass die allgemeinen sozialen, politischen und anderen Vorteile, die Angehörige europäischer Staaten in Kolonialgebieten besitzen, auch Schweizern zugute kommen $\ll^{16}$. In diesem Zusammenhang sprach er von der Schweiz als »lachende Dritte«: »Es kann kein Zweifel darüber bestehen, dass die Schweiz auf diese Weise als >tertius gaudens $<$ aus dem Imperialismus der andern gerade als nichtimperialistisches Land Nutzen zieht. $\ll^{77}$ Gerade weil die Schweiz keine formale Kolonialmacht war, konnten Schweizer Firmen nach der Dekolonisierung erfolgreich ihre Stellung sichern und dabei als »unverdächtiger« Partner gegenüber den ehemaligen Kolonien auftreten. Es wäre interessant zu erforschen, ob der Wirtschaftsaufschwung, den die Schweiz nach dem Zweiten Weltkrieg erlebte, auch auf diese vorteilhafte Positionierung zurückzuführen ist. Rentable Großprojekte, technisches Knowhow und Entwicklungshilfe wurden in dieser Zeit jedenfalls gewinnbringend zusammengebracht. ${ }^{18}$

Parallel zu dieser Entwicklung wurde insbesondere seit Beginn der 1970er Jahre Kritik an den Verflechtungen von Schweizer Unternehmen mit neokolonialen globalen Wirtschaftsstrukturen laut. Tiersmondistische Bewegungen oder Organisationen wie die Erklürung von Bern, gegründet 1968, oder die Aktion Finanzplatz Schweiz, die 1978 ihre Arbeit aufnahm, machen seit Jahrzehnten auf die vielfältigen Verbindungen zwischen dem Schweizer Außenhandel und diktatorischen, korrupten Regimes aufmerksam sowie auf die Geschäftspraktiken transnationaler Konzerne, welche gegen die Menschenrechte verstoßen oder arbeitsrechtliche Minimalstandards unterlaufen. ${ }^{19}$ Zentral für diese Widerstandsbewegungen war das

14 | Siehe dazu beispielsweise David/Bouda/Schaufelbuehl, Schwarze Geschäfte; Fässler, Reise ins Schwarz-Weiss und Stettler/Haenger/Labhardt, Sklaverei und Kredite. Vgl. zur Debatte auch Kuhn/Ziegler, Schweiz und die Sklaverei.

15 | Behrendt, Die Schweiz und der Imperialismus.

16 | Ebd., S. 51.

17 | Ebd., S. 46.

18 | Siehe dazu die hier publizierten Artikel von Sara Elmer und Daniel Speich.

19 | Kalt, "Nestlé«; Kalt, Tiersmondismus; Kuhn, Entwicklungspolitische Solidarität. Vgl. auch den Beitrag von Konrad Kuhn in diesem Band. 
Engagement gegen das südafrikanische Apartheidsregime. ${ }^{20}$ Schweizer Firmen tätigten lukrative Geschäfte in Südafrika; die offizielle Schweiz konnte sich nicht zu einem Boykott durchringen. ${ }^{21}$ Noch heute sind diesbezüglich Akten aufgrund einer speziell verlängerten Schutzfrist im Bundesarchiv unter Verschluss, was eine notwendige Aufarbeitung der Beziehungen zwischen der Schweiz und Südafrika auch auf Druck der Rüstungsindustrie weiterhin verunmöglicht. ${ }^{22}$

Der vorliegende Sammelband beschäftigt sich allerdings nicht primär mit finanziellen Transaktionen zwischen Schweizer Geschäftsleuten und kolonialen Handelsfirmen, Aktienbeteiligungen an Sklavenschiffen oder den wirtschaftlichen Folgen des Kolonialismus in der neokolonialen Ära. Sein Fokus liegt auf den kulturellen Aspekten des Kolonialismus (und somit auf den diskursiven, semantischen und imaginären Ausformungen kolonialer Projekte) und seinen Nachwirkungen bis hinein in die Gegenwart. Aufgrund dieser Ausrichtung liegt es auf der Hand, dass die postcolonial studies, die seit über 30 Jahren und vornehmlich im englischsprachigen Raum betrieben werden, einen zentralen Anknüpfungspunkt für unsere Forschung bieten.

Der Begriff des Postkolonialen führt, wie die Kulturwissenschaftlerin Doris Bachmann-Medick anmerkt, zwei Bedeutungsstränge zusammen, die ein anhaltendes Spannungsverhältnis erzeugen. ${ }^{23}$ Als eine kritische historische Kategorie bezeichne »postkolonial« einerseits die nachhaltige Prägung der globalen Situation durch Kolonialismus, Dekolonisierung und neokolonialistische Tendenzen. Andererseits werde über diese historische Verortung hinaus eine diskurskritische Kulturtheorie angestoßen, die den Eurozentrismus gängiger Wissensordnungen und Repräsentationssysteme kritisiert und oft einen Gegenwartsbezug aufweist. Daher werden die postcolonial studies auch immer relevanter für Länder, die durch die Beschäftigung mit den kulturellen Aspekten des Rassismus die kolonialen Bestandteile ihrer eigenen Geschichte entdecken. Das Präfix post bezieht sich dann nicht einfach temporal auf die Zeit nach der Aufösung der Kolonialreiche, sondern verweist auf das Weiterwirken kolonialistischer Strukturen in neuen, insbesondere kulturellen Formen. Hierin ist ein wesentlicher Unterschied zum Begriff des Neokolonialismus zu verorten: Während dieser zumindest in seiner ursprünglichen Bedeutung ein ökonomisches Konzept darstellte sowie die neuen Aspekte

20 | Kreis, Die Schweiz und Südafrika, S. 139.

21 | Die internationalen Sanktionen hatten nicht nur ökonomische, sondern auch politische Auswirkungen: Sie führten dazu, dass sich die südafrikanische Wirtschaftselite schließlich vom Apartheidsregime distanzierte. "Die Schweiz leistete an diesem Prozess keinen Beitrag", hält Georg Kreis fest (ebd., S. 496). Vgl. auch http://www.apartheidreparations.ch.

22 | Siehe dazu das Interview von Jan Jirát mit Sacha Zala auf http://www.woz.ch/ artikel/2011/nr35/kultur \%20_\%20wissen/21094.html, 13.09.2011.

23 | Siehe für die nachfolgenden Überlegungen Bachmann-Medick, Cultural Turns, S. $184 \mathrm{ff}$. 
eines »Kolonialismus nach der Dekolonisation« betont, stehen in postkolonialen Theorien spätestens seit der Veröffentlichung von Edward Saids Werk Orientalism im Jahre 1978 die visuellen und diskursiven Repräsentationen, vor allem mit Bezug auf die Kontinuität von Rassismen und Ethnozentrismen, im Vordergrund.

Mit dem Aufkommen der postcolonial studies in den 1980er Jahren erfährt der Begriff postkolonial somit eine Bedeutungsverschiebung: Er mutiert von einem imperialismuskritischen historischen Epochenbegriff $\mathrm{zu}$ einem politischprogrammatischen und diskurskritischen Konzept. ${ }^{24}$ Es gilt fortan, ein kritisches Analyseinstrumentarium zu entwickeln, mit dem die anhaltenden und weiterhin problematischen Konstruktionen des »Anderen« sowie seine grundlegende Bedeutung für die Herstellung des (westlichen, weißen) Subjekts analysiert und aufgearbeitet werden können. Für die Konstitution europäischer Subjektivitäten und nationaler Identitäten, so zeigen die postcolonial studies, spielen die Abgrenzung vom kolonialen »Anderen«, aber auch seine Vereinnahmung und Instrumentalisierung eine grundlegende Rolle. ${ }^{25}$ In diesem Kontext wird sowohl auf die diskursive Gewalt dieses othering hingewiesen als auch versucht, andere Repräsentationen und vor allem Selbstrepräsentationen bisher marginalisierter Gesellschaften und ethnischer Gruppen zu ermöglichen, ohne allerdings erneut dem Mythos der Authentizität zu verfallen. Aus feministischer und queerer Perspektive wird die Frage nach der Artikulationsfähigkeit, Selbstrepräsentation und Handlungsfähigkeit des postkolonialen Subjekts zudem mit der grundlegenden Bedeutung von Geschlecht und Sexualität verknüpft. Dabei wird deutlich, dass die globale Arbeitsteilung nicht nur postkolonial strukuriert, sondern auch vergeschlechtlicht und heteronormativ kodiert ist. $^{26}$

Ohne den Anspruch auf Vollständigkeit erheben zu wollen, sollen im Folgenden einige grundlegende Erkenntnisse der postkolonialen Agenda skizziert werden. ${ }^{27}$ Die postkoloniale Theorie vermittelt Einsichten in die Dynamik grenz-

24 Bereits die Generation von Intellektuellen wie Frantz Fanon, Kwame Nkrumah oder Aimé Césaire setzte eine selbstkritische "Deplatzierung" des europäischen Theoriediskurses in Gang. Die erlangte Einsicht, dass koloniale Macht nicht nur ökonomisch, sondern auch diskursiv über das (westliche) Wissenssystem ausgeübt wurde, hatte weitreichende Folgen. Für einen allgemeinen Überblick über "Theorien westlicher Hegemonie" siehe Wolfe, "History and Imperialism".

25 | Vgl. Trinh, Woman, Native, Other; Hall, "The Spectacle of the 'Other'".

26 | Zur Frage der Feminisierung globaler Armut und Arbeitsteilung siehe beispielsweise Spivak, In Other Worlds oder Can the Subaltern.

27 | Siehe beispielhaft für den englischsprachigen Raum: Young, Postcolonialism; Schwarz/Ray, A Companion to Postcolonial Studies. Für den deutschsprachigen Raum: Castro Varela/Dhawan, Postkoloniale Theorie; Conrad/Randeria, Jenseits des Eurozentrismus; Fischer-Tiné, "Postkoloniale Studien «; Lindner, "Neuere Kolonialgeschichte". Ebenso die von Patricia Purtschert formulierten Thesen in "Postkoloniale Diskurse". Das Folgende bezieht sich vor allem auch auf Conrad/Eckert/Freitag, Globalgeschichte, S. 22-24. 
überschreitender Austauschprozesse. Entgegen der klassischen Modernisierungstheorie ${ }^{28}$, die von einem makrohistorischen Modell der Diffusion und Adaption beziehungsweise von einer »Verwestlichung « der Welt ausgeht, betont sie das komplexe Geflecht von agency, lokal spezifischen Aneignungsstrategien und strategischen Modifikationen sowie die Bedeutung von Hybridisierung als Korrektiv zu einem Denken, das von klar abgegrenzten Nationen, Völkern oder »Rassen« ausgeht. ${ }^{29}$ Kultur wird derart nicht im Sinne einer »Container-Theorie« als statische Entität mit fest umrissenen, nationalen, ethnischen und regionalen Grenzen gedacht. Vielmehr gelten die Verflechtungszusammenhänge der modernen Welt als Ausgangspunkt einer transnationalen Geschichtsschreibung. Geschichte im Sinne von entangled histories zu konzeptualisieren bedeutet, die Abhängigkeiten, Überlagerungen und Interdependenzen im Kontext der Machtasymmetrien der modernen Welt zu untersuchen. ${ }^{30}$

Die postkoloniale Theorie formuliert Zweifel am Eurozentrismus, also der westlichen Deutungshegemonie und der Vorstellung, die Moderne sei europäischen Ursprungs, und unterzieht Begriffe wie Moderne oder Aufklärung einer radikalen Kritik. ${ }^{31}$ Denn wenn es um die Geschichte der nicht westlichen Welt geht, so kritisiert die postkoloniale Theorie, werden beständig die Tropen der »Unvollständigkeit« oder der »Unzulänglichkeit« zum Einsatz gebracht. Damit, schreibt Dipesh Chakrabarty, wird impliziert, dass die außereuropäische Welt den »Warteraum der Geschichte erst durch eine »nachholende Modernisierung « verlassen könne. ${ }^{32}$ Andererseits betont er, dass gerade die Kritik am Kolonialismus nicht unabhängig davon gedacht werden kann, wie das Erbe der europäischen Aufklärung in den Kolonien angeeignet wurde - denn »[p]ostcolonial scholarship is committed, almost by definition, to engaging universals - such as the abstract figure of the human or that of Reason - that were forged in the eighteenth-century Europe and that underlie the human sciences. «33 Postkoloniale Ansätze zielen auf die Überwindung des Tunnelblicks ${ }^{34}$, der die Geschichte Europas aus sich heraus erklärt. Ziel ist die Problematisierung der eurozentrischen Fundamente und jener Marginalisierungen, welche die Moderne erst ermöglicht haben. Es findet damit eine neue Einschätzung der Ursprünge der Moderne und vor allem der Vorstellungen von »Rasse« statt - oder wie es Barbara Weinstein ausdrückt: »[T]he concept of race is not just the >barbaric underside of modernity [... I]t is the very face of the modernity that emerges in the context of Europe's >civilizing mission « ${ }^{35}$.

28 | Vgl. Bhambra, Rethinking Modernity; Mergel, Modernisierung.

29 | Vgl. zum Beispiel Bhabha, Verortung.

30 | Vgl. Lepenies, Entangled Histories. Siehe auch Comaroff/Comaroff, Ethnography.

31 | Zum Begriff des Eurozentrismus vgl. Dussel, "Beyond Eurocentrism".

32 | Chakrabarty, Provincializing Europe, S. 9.

33 | Ebd., S. 5.

34 | Tanner, "Tunnelblick".

35 | Weinstein, "History Without a Cause?", S. 91. 
Die Beschäftigung mit postkolonialen Ansätzen sensibilisiert somit nicht nur für die (oftmals gewaltbeladenen) politischen, kulturellen, wirtschaftlichen und sprachlichen Transformationen, welche die von Europa kolonisierten Gesellschaften erfuhren, sondern sie stärkt das Bewusstsein dafür, dass auch europäische Gesellschaften durch die koloniale Interaktion und durch die Rückwirkungen der kolonialen Erfahrung geprägt und teilweise hervorgebracht worden sind. Der Kolonialismus betrifft auf unterschiedliche Weise die ganze Welt, wie es Andreas Eckert und Shalini Randeria formuliert haben: »Wir leben alle in einer postkolonialen Welt, nicht nur jene Menschen in und aus ehemals kolonisierten Gebieten ${ }^{36}{ }^{6}$. Der in den postcolonial studies verwendete Begriff der kolonialen Moderne deutet darauf hin, dass die Herausbildung moderner Kategorien, Strukturen, Reformen und Institutionen auf grundsätzliche Weise mit den kolonialen Kontexten korreliert, in denen sie sich vollzog. Sebastian Conrad zeigt beispielsweise auf, wie in den deutschen Kolonien seit der Mitte der 188oer Jahre die »Arbeiterfrage« zu einem dringlichen Problem der Kolonialpolitik wurde. Das Projekt der »Erziehung zur Arbeit« fokussierte ebenso auf die Bewohnerinnen und Bewohner der Kolonien wie auf die sogenannten Vagabunden und Arbeitsscheuen Europas. Einen ähnlichen Befund liefern Harald Fischer-Tinés Untersuchungen zum Engagement der Heilsarmee in Großbritannien und Britisch-Indien. ${ }^{37}$ Er zeigt, wie in Indien gegen Ende des 19. und zu Beginn des 20. Jahrhunderts Gesetze verabschiedet wurden, um die nomadischen »criminal tribes « in abgeschlossene und bewachte Reservate zu befördern und sie in Arbeitslagern zu einem sesshaften Lebensstil zu zwingen. Gleichzeitig wurden Arbeitshäuser für weiße Landstreicher errichtet, um sie für die indische Bevölkerung weitgehend unsichtbar $\mathrm{zu}$ machen, denn die Existenz eines weißen Lumpenproletariats verwischte die Grenze zwischen Kolonialisierten und Kolonisierenden auf unerwünschte Weise. Das »Wissen«, das im Umgang mit diesen Gruppen in Indien produziert wurde - beispielsweise von der Heilsarmee, die mit der Führung dieser Heime beauftragt war -, zirkulierte und kam so auch nach Europa zurück. ${ }^{8}$ Solche Phänomene miteinander zu verbinden macht, so Sebastian Conrad, die »Verflechtung zweier Praxisformen« deutlich, »die aus einer sozialreformerischen Politik eine Praxis der Exklusion werden ließen «39.

36 | Eckert/Randeria, "Geteilte Globalisierung", S. 11.

37 | Vgl u.a. Fischer-Tiné, "Reclaiming savages"; Fischer-Tiné, Low and Licentious Europeans.

38 | Francesca Falks Ausführungen zu den Fahrenden in diesem Band zeigen, was diese Verhältnisse wiederum mit der Schweiz zu tun haben. Thematisiert wird dies auch in Falk, Eine gestische Geschichte der Grenze, S. 108-111. Bernhard Schär verweist ebenfalls auf die Verbindung zwischen inner- und außereuropäischen Formen des othering, dazu Schär, "Mariella Mehr".

39 | Conrad, Globalisierung und Nation, S. 28 und Kapitel 2. 
Methodisch erfordert das, Metropole und Kolonie nicht als getrennte Bereiche zu behandeln, sondern sie innerhalb eines analytischen Rahmens zu untersuchen. ${ }^{\circ}{ }^{\circ}$

\section{EUROPÄISCHE ERBSCHAFT}

Wo steht die Diskussion um das koloniale Erbe in anderen westeuropäischen Staaten? ${ }^{41}$ Die Thematisierung der kolonialen Vergangenheit ist in Großbritannien sowohl in der Forschung wie auch in der Öffentlichkeit wohl am stärksten präsent und deutlicher sichtbar als in anderen Staaten. ${ }^{42}$ Dennoch wurden die Kolonien lange Zeit als etwas »Außenstehendes « im Verhältnis zur eigenen nationalen Geschichte thematisiert, wie Frederick Cooper anmerkte. ${ }^{43}$ Die damit verknüpfte Schuld und die Trauer um das verloren gegangene Weltreich würden bis zum heutigen Tag verdrängt. ${ }^{44}$ In Frankreich wiederum ist, wie bereits erwähnt, eine postkoloniale Perspektive auf die eigene Geschichte und Gegenwart keine Selbstverständlichkeit. ${ }^{45}$ Erstaunlich ist die lange ausbleibende Rezeption postkolonialer Perspektiven in Frankreich insofern, als einige der wichtigsten Vorreiter der postkolonialen Theorie, etwa Léopold Sédar Senghor, Frantz Fanon, Albert Memmi oder Aimé Césaire, ihre Analysen im Kontext des französischen Kolonialregimes entwickelt haben. ${ }^{4}$ Nach einer längeren Zeit akademischer Marginalisierung kehren diese Theorien, vermittelt vor allem durch US-amerikanische Adaptionen im Kontext der postcolonial studies, wieder nach Frankreich zurück - eine Bewegung, die wiederum kritische Fragen bezüglich hegemonialer Strukturen im Wissenschaftsbetrieb aufwirft. 47

Die verstärkte Diskussion postkolonialer Themen ereignet sich in Frankreich paradoxerweise in einem politischen Klima, in dem die Forderung, den Kolonialismus und das damit verbundene schlechte Gewissen Frankreichs endlich ad acta

40 | Vgl. dazu Stoler/Cooper, "Between Metropole“.

41 | Vgl. dazu auch Ponzanesi/Blaagard, Deconstructing Europe; Poddar/Patke/Jensen, A Historical Companion.

42 | Das britische Empire war die größte Kolonialmacht in der Geschichte. Es umfasste Dominions, Kronkolonien, Protektorate, Mandatsgebiete und andere abhängige Gebiete. Dazu gehörten u.a. Gebiete in Asien, Afrika und Ozeanien.

43 | Cooper, Colonialism, S. 171.

44 | Eckert, "Der Kolonialismus".

45 | Im 19. Jahrhundert war Frankreich die zweitgrößte Kolonialmacht der Welt und besaß vor allem Gebiete in Afrika und Asien. Für einen aktuellen Überblick über die Rezeption postkolonialer Ansätze in Frankreich vgl. Mbembe, "Provincializing France?".

46 | Zudem beziehen sich postkoloniale Ansätze ganz wesentlich auf die Arbeiten französischer Intellektueller wie Jacques Derrida, Michel Foucault oder Gilles Deleuze.

47 | Siehe beispielsweise Ahmad, In Theory. 
zu legen, mehrheitsfähig geworden ist. ${ }^{4}$ So löste ein 2005 verabschiedetes Gesetz, das die positiven Auswirkungen der kolonialen Aktivitäten Frankreichs betonte, heftige Debatten aus. ${ }^{49}$ Kurz darauf kam mit Nicolas Sarkozy ein Wortführer dieses neuen Selbstverständnisses an die Macht. Notorische Berühmtheit erlangte er mit seiner Dakar-Rede vom Juli 2007, in der er Afrika als geschichtslos beschrieb und dem Kontinent seine Hilfe beim Eintritt in die Moderne anbot. Achille Mbembe hielt daraufhin fest:

"Contrairement à la génération des 'Papa-Commandant‘ (de Gaulle, Pompidou, Giscard d'Estaing, Mitterrand ou Chirac) qui épousait tacitement le même préjugé tout en évitant de heurter de front leurs interlocuteurs, les 'nouvelles élites، de France estiment désormais qu'à des sociétés aussi plongées dans la nuit de l'enfance, l'on ne peut s'adresser qu'en s'exprimant sans frein, dans une sorte de vierge énergie.“50

Mbembe macht den relevanten Bruch somit nicht in der Haltung des Präsidenten aus, dessen Vorgänger die kolonialen Vorurteile stillschweigend weitergeführt haben, sondern in dessen Stil: Rassistisches Denken ist (wieder) auf höchstem politischen Niveau salonfähig geworden. Dem steht nicht nur die wachsende Bedeutung postkolonialer Forschung an den Universitäten entgegen ${ }^{51}$, sondern auch neuere politische Bewegungen wie die Indigènes de la République, welche die Rassismen in der hegemonialen Vorstellung der französischen Nation aufgreifen, karikieren und anfechten..$^{2}$

Im Unterschied zur aktuellen französischen Regierung, welche die positiven Aspekte und Auswirkungen des Kolonialismus betont sehen möchte, dominiert in Deutschland in Politik und Öffentlichkeit die Tendenz, die eigene Kolonialgeschichte als unbedeutende Randnotiz der Geschichte abzutun. ${ }^{53}$ So wurde die 2001 gegründete International Security Assistance Force (ISAF) in Afghanistan, an der deutsche Soldaten partizipieren, von den Medien als »Schutztruppe« bezeichnet. Dies geschah, wie Andreas Eckert und Albert Wirz festhalten, »ungeachtet der Tat-

48 Auffallend ist bei diesen neueren Diskussionen über Kolonialismus und Rassismus, dass die biologistischen Fundierungen von Rassismus häufig einer kulturalistischen Argumentation weichen. Vgl. dazu Balibar/Wallerstein, Rasse, Klasse, Nation.

49 | Vgl. Bancel, "Introduction", S. 25. Ebenso Blanchard/Bancel/Lemaire, Culture coIoniale und Mollenhauer, "Erinnerungspolitik". Aufgrund der vehementen Kritik entschied sich der damalige Präsident Jacques Chirac kurz darauf dazu, die Passage wieder aus dem Gesetzestext zu entfernen.

50 | Mbembe, "L'afrique". Vgl. auch Gassama et al., L'Afrique répond.

51 | Vgl. etwa Dorlin, Sexe, race, classe; Fassin, Question Sociale; Blanchard/Bancel/Lemaire, Culture Coloniale; Bancel et al., Ruptures Postcoloniales.

$\mathbf{5 2}$ | Vgl. dazu die Webseite http://www.indigenes-republique.fr, 24.03.2011.

53 | Vgl. Krüger, "Vergessene Kriege". Deutschland besaß im 19. Jahrhundert vor allem in Afrika und der Südsee Kolonien und sogenannte Schutzgebiete. 
sache, dass der Begriff die deutsche Kolonialarmee bezeichnete, jene Truppe also, welche die innere Eroberung der deutschen Kolonien erzwang und [...] im Namen des Kaisers, des Fortschritts und des Deutschen Reichs genozidäre Kriege in Afrika führte «54. Entsprechend dieser Amnesie gegenüber der eigenen Kolonialgeschichte wird davon ausgegangen, so Hito Steyerl und Encarnacion Gutiérrez Rodríguez, dass das postkoloniale Machtverhältnis »in Deutschland nicht existiert oder irrelevant sei oder Vorgänge beschreibe, die woanders stattfinden «55. Verbunden mit der Weigerung, die eigene Kolonialgeschichte zu bearbeiten, ist auch der Widerstand gegen Theorien, die ihren Fokus auf das »Weißsein« richten, dem gleichsam blinden Fleck und Zentrum von Rassifizierungsdiskursen. Es herrsche, so schreibt Maureen Maish Eggers, »offensichtlich ein großer Widerstand gegen eine explizite Kennzeichnung und damit Markierung von Weißsein in Deutschland « ${ }^{56}$.

Trotz solcher Widerstände haben sich Kolonialgeschichte, postcolonial und whiteness studies in der letzten Dekade vermehrt als populäres Thema in den Kultur- und Geschichtswissenschaften an deutschsprachigen Hochschulen etabliert. ${ }^{57}$ Hannah Arendts Monumentalwerk Elemente und Ursprünge totaler Herrschaft, welches mögliche Verbindungen zwischen dem Kolonialismus und der Shoa erörtert, stellt dabei immer wieder einen wichtigen Anknüpfungspunkt dar. ${ }^{5}$ In jüngerer Zeit ist zudem vermehrt die Art und Weise, in der rassistische Inhalte mit der aktuellen deutschen Alltagskultur verknüpft sind, in den Blick gerückt. Programmatisch hält Antje Schuhman dazu fest: »Visible, yet often not recognized as such, colonial images, language and concepts re-inscribe white superiority on a dayto-day basis into German politics, society and popular culture and are, as such, part of our collective stereotype reservoir.«59 Kien Nghi Ha wiederum weist auf

54 | Eckert/Wirz, "Wir nicht", S. 373.

55 | Steyerl/Gutiérrez Rodríguez, "Einleitung", S. 8.

56 | Eggers, "Schwarzes Wissensarchiv", S. 19.

57 | In der letzten Dekade sind eine ganze Reihe Untersuchungen zu dem Thema entstanden bzw. sind im Entstehen begriffen. Siehe exemplarisch Conrad/Osterhammel, Das Kaiserreich; Friederichsmeyer/Lennox/Zantop, Imperialist Imagination; Hell/Steinmetz, "Visual Archive «; Kundrus, Phantasiereiche; Laak, Über alles in der Welt; Honold/Scherpe, Mit Deutschland um die Welt; Maß, Weiße Helden; Steinmetz, "Decolonizing“; Zimmerer/ Zeller, Völkermord; Walgenbach, Die weiße Frau; Steyerl/Gutiérrez Rodríguez, Spricht die Subalterne deutsch?; Eggers et al., Mythen, Masken; Dietze/Brunner/Wenzel, Kritik des Okzidentalismus; Ha et al., re/visionen.

58 | Vgl. Arendt, Elemente und Ursprünge. Ebenso Gerwarth/Malinowski „Der Holocaust“; Grosse, "German Colonialism «; Kundrus, "Kolonialismus, Imperialismus" und Zimmerer, "Geburt des 'Ostlandes"

59 | Schuhmann, "Exoticizing the Erotic", S. 108. Schuhmann verbindet diese verdrängte und mit Nostalgie aufgeladene deutsche Kolonialgeschichte beispielsweise mit der Werbung eines deutschen Möbelhauses, das seine Produkte in einer "Colonial Style-Sonderschau“ an den Mann und an die Frau zu bringen sucht. 
die verdrängten kolonialen Aspekte der deutschen Arbeitsmigrationspolitik hin. ${ }^{60}$ So werde deren Beginn gemeinhin im Jahre 1955 mit dem Abschluss des Anwerbeabkommens mit Italien festgesetzt. Ausgeblendet bleibt in einer solchen Sicht die osteuropäische Arbeitsmigration Preußens, die ab 1890 im großen Stil einsetzte und Konturen einer »inneren Kolonialisierung « aufweist. Ha zufolge wurde die »Struktur wie die Zielsetzung der deutschen Arbeitsmigrationspolitik [...] grundlegend durch ihren gesellschaftlichen Entstehungskontext im Zeitalter des Imperialismus geformt « ${ }^{61}$. Durch die Thematisierung dieser Verwobenheit könne die lange Genealogie der »rassifizierten Stratifikation« aufgezeigt werden, die den Umgang mit den sogenannten Gastarbeitern kennzeichnet und auch die aktuelle Migrationspolitik charakterisiert.

In einem weiteren Nachbarland der Schweiz, in Italien, ist ein langandauerndes Fehlen einer postkolonialen Perspektive ebenfalls augenfällig. ${ }^{62}$ So Cristina Lombardi-Diop und Caterina Romeo:

"Although historians and cultural critics have been documenting the colonial experience and its possible repercussions on contemporary Italy since the 1980 s, no public or academic debate has developed which openly and critically confronts the colonial past, its removal from Italy's public memory, and its lingering legacy." ${ }^{63}$

Auch Sandro Mezzadra spricht von einer »tardiva ricezione italiana degli studi postcoloniali ${ }^{64}$. In den Schulen und im Fernsehen ist die eigene Kolonialgeschichte kaum präsent. ${ }^{65}$ Exaußenminister Gianfranco Fini, dem es gelungen war, die neofaschistische Bewegung Movimento Sociale Italiano in die Regierungspartei Alleanza Nazionale zu »verwandeln«, bemerkte 2006 während einer migrationspolitischen Veranstaltung, Europa sei in den Kolonien ein großes Element der Zivilisierung gewesen und nicht alle Aspekte des Kolonialismus seien negativ zu bewerten. Wenn man sich vor Augen führe, so Fini, wie heruntergekommen Äthiopien, Somalia und Libyen heute seien und wie sich ihr Zustand unter italienischer Kolonialherrschaft präsentiert habe, müsse dies in den Ländern selber Anlass für eine Neubewertung der italienischen Rolle sein. ${ }^{66}$ In einem postkolonialen Zusammenhang ist auch das 2009 abgeschlossene Abkommen zwischen Libyen und

60 | Ha, "Koloniale Arbeitsmigrationspolitik".

61 | Ebd., S. 66.

62 I Italien besaß vor allem in Nord- und Ostafrika Kolonien, wozu beispielsweise Libyen und Äthiopien gehörten.

63 | Romeo, "Postcolonial Italy". Für ein aktuelles Beispiel postkolonialer Forschungsansätze im italienischen Kontext siehe auch Amodeo, "In the Empire's Eyes" sowie Triulzi, "Dispacing the Colonial Event".

64 | Mezzadra, La condizione.

65 | Mattioli, "Viva Mussolini!".

66 | Zitiert nach Mattioli, ebd., S. 76. 
Italien zu sehen. Damals erhielt Libyen eine Entschädigung für die erlittene Kolonialisierung zugesprochen (5 Milliarden Dollar in 20 Jahren) und verpflichtete sich im Gegenzug, afrikanische Migrantinnen und Migranten abzuwehren:

"Mit der Reparationszahlung sollen eine 1'600 Kilometer lange Küstenautobahn, die das Land von der ägyptischen zur tunesischen Grenze durchzieht, und weitere Infrastrukturprojekte realisiert werden. Darüber hinaus sieht das Vertragswerk eine enge Zusammenarbeit auf den Gebieten von Wirtschaft, Handel und Immigrationsabwehr vor. Libyen versprach, Italien privilegiert bei Mineralöl- und Erdgaslieferungen zu behandeln. Von den Bauprojekten sollen dereinst auch italienische Firmen profitieren. " ${ }^{67}$

Als Ironie der Geschichte mag der Umstand gelten, dass Straßenbau auch während des italienischen Kolonialismus das wichtigste Investitionsprojekt war. Inzwischen haben die revolutionären Ereignisse das Abkommen allerdings überholt. Das Beispiel Italien zeigt: Die europäische Illegalisierung der Immigration ist durch ihre koloniale Vorgeschichte zu verstehen. Alltagsrassismen, die auch aus Kolonialzeiten stammen, prägen heute noch den Umgang mit Migrantinnen und Migranten in Italien, und zwar auch von jenen, die nicht aus italienischen Kolonialgebieten kommen. Dies zeige, so Jacqueline Andall und Derek Duncan, »that colonialism is embedded in the contemporary ${ }^{68}$.

Im letzten Jahrzehnt entwickelten sich interdisziplinäre Forschungsprojekte, die postkoloniale Perspektiven auf die skandinavischen Länder - insbesondere Schweden, Norwegen, Finnland und Dänemark - richteten. Damit wurde der theoretische Fokus der postkolonialen Theorie weg von den Kulturen und Gesellschaften der ehemaligen formalen Kolonien hin zu den Ländern gelenkt, die sich explizit als Außenseiter innerhalb der europäisch-kolonialen Machtkonstellation verstanden. Das Augenmerk richtete sich zuvorderst auf die Frage, inwiefern und auf welche Art und Weise diese Länder in Vergangenheit und Gegenwart kulturell und wirtschaftlich von kolonialen Beziehungen geprägt wurden und sind. ${ }^{6}$ In diesem Zusammenhang entwickelte eine Gruppe feministisch orientierter Wissenschaftlerinnen das Konzept der colonial complicity. ${ }^{70}$ Die skandinavischen Länder, so wird damit gezeigt, »ha[ve] neither been historically situated as one of the colo-

67 | Ebd., S. 140.

68 | Andall/Duncan, "Memories", S. 21.

69 | Auch in Bezug auf den ehemaligen Vielvölkerstaat der Habsburger hat im letzten Jahrzehnt die Anwendung von postkolonialen Ansätzen zu einer neuen Wertung dieser Geschichte geführt. Auch wenn die Habsburgermonarchie keinen Kolonialstaat im engeren Sinn darstellte, können die Theoriebildungen der postcolonial studies zur Erforschung der österreichischen Imperialgeschichte sinnvoll angewendet werden. Vgl. Feichtinger/ Prutsch/Csaky, Habsburg Postcolonial. Zur postkolonialen Situation in Österreich vgl. auch Johnston-Arthur, "'Es ist Zeit‘،. Zum Baltikum vgl. Kelertas, Baltic Postcolonialism.

70 | Siehe dazu Keskinen et al., Complying. 
nial centres in Europe nor ha[ve they] been an sinnocent victim< or mere outsider of the colonial projects «71. Der Begriff der Komplizität bedeutet in einer postkolonialen Leseart die Beteiligung an den hegemonialen Diskursen des Westens ebenso wie an deren universalistischen Denkmustern und Praktiken der Herrschaft. Gerade für Länder außerhalb der westlichen Zentren, so Ulla Vuorela, stellt Komplizität eine Art und Weise dar, näher an das Vorbild dieser Machtzentren zu rücken und »dazugehören « zu wollen. Für das Beispiel Finnland bedeutete dies beispielsweise die Verdrängung der indigenen Bevölkerung der Sami aus ihrem ehemaligen Lebensraum im Süden des Landes, also ein Prozess der »internen Kolonisation«. Ebenso wurde von Finnland zu Beginn des 20. Jahrhunderts die Kolonisierung des heutigen Namibia, wo finnische Missionsgesellschaften aktiv waren, angedacht, doch schließlich verworfen. Aber auch jenseits der konkreten kolonialen Eroberungen und Vertreibungen kann über Wissensordnungen eine »universale Wahrheit« zur Legitimation von Expansionen oder zur Rechtfertigung gewisser Lebensstile eingesetzt werden. Wenn sie auch nicht direkt an den europäisch-kolonialen Eroberungen beteiligt waren, so existierten und existieren noch immer verschiedene Anknüpfungspunkte der skandinavischen Länder an die Art von Wissen, welche im Kontext oder gar in Unterstützung von kolonialen Projekten entstand. ${ }^{72}$ Ein konkretes Beispiel dieser Wissensproduktion kann für Finnland bereits zu Beginn des 20. Jahrhunderts in der Beteiligung von finnischen Ethnologinnen und Ethnologen an der Entwicklung von Theorien ausgemacht werden, die die Menschheit in evolutionäre Stufen unterteilte und spezifisches Wissen für die kolonialen Administrationen bereitstellten. Parallelen können für die neuere Zeit auch in paternalistisch-rassistischen Praktiken und Kommunikationsweisen der finnischen Entwicklungshilfe und der Anpassung an neoliberale Bedingungen westlicher Finanzinstitute (Weltbank und Internationaler Währungsfond) ausgemacht werden. ${ }^{73}$ Für Schweden wurden Schulbücher auf klassische Stereotypen in Bezug auf Afrika untersucht. Dabei wurden bis in die jüngere Zeit Vorstellungen von den »höheren « und »niederen« »Rassen«, den »kriegerischen afrikanischen Stämmen«, des »geschichtslosen « und exotischen Afrika, den Europäern als »Zivilisatoren « und anderen Bildern festgemacht - Bilder, die den meisten westeuropäischen Ländern in Schulbüchern, Massenmedien und Populärkultur gemeinsam sind. ${ }^{74}$ Viele der skandinavischen Länder, so das Fazit der Autorinnen, profitierten wirtschaftlich vom Sklavenhandel, verbreiteten über missionarische Aktivitäten die Idee der christlichen und westlichen Überlegenheit und waren an kolonialen Unterneh-

71 | Vuorela, "Colonial Complicity“, S. 19. Eine ähnliche Perspektive entwickelt Kristín Loftsdóttir mit Blick auf Island, vgl. Loftsdóttir, "Negotiating White Icelandic Identity" und Loftsdóttir, "The Loss of Innocence. The Icelandic Financial Crisis and Colonial Past". Vgl. auch Gullestad, "Normalising Racial Boundaries".

72 | Vuorela, "Colonial Complicity", S. 21.

73 | Ebd. Vgl. dazu auch Eriksson Baaz, Paternalism of Partnership.

74 | Vgl. Palmberg, "Nordic Colonial Mind". 
men direkt oder indirekt beteiligt. Hier zeigen sich frappante Ähnlichkeiten zur Schweizer Konstellation. ${ }^{75}$ Nicht zufällig forderte der Nationalrat Josef Lang in einer Motion vom 19. Dezember 2006, die Schweiz solle gemeinsam mit anderen » kleineren Sklaverei- und Kolonialnationen«« wie »Schweden, Dänemark und Deutschland (Brandenburg-Preußen) « eine Initiative zur »Aufarbeitung und Wiedergutmachung von Sklaverei und Sklavenhandel « ergreifen. ${ }^{76}$

\section{"KOLONIALE KOMPLIZENSCHAFT", "HEIMLICHES IMPERIUM “ ODER "POSTKOLONIALE SCHWEIZ«?}

Ist es sinnvoll, auch in Bezug auf die Schweiz von einer »kolonialen Komplizenschaft « zu sprechen? Oder bedarf es eines anderen Vokabulars? Welcher Leitbegriff bietet sich an, um die vielfältigen Verwicklungen der Schweiz mit den kolonialen Regimes und ihren postkolonialen Effekten zu untersuchen? Müsste man von Schweizer Akteurinnen und Unternehmern als Handlanger, Gehilfinnen und Kollaborateure, Profiteurinnen und Trittbrettfahrer des Kolonialismus sprechen? Oder wäre es sinnvoller, die in den $1980 e r$ Jahren entwickelte feministische Debatte um die Mittäterinnenschaft aufzugreifen?77 Die historische Forschung, die sich mit den kolonialen und nachkolonialen Schweizer Aktivitäten befasst, verwendet oftmals den Begriff des Imperialismus. So teilt mit Behrendt auch Beat Witschi, der die schweizerischen Handelsbeziehungen mit der Levante untersucht hat, die Einschätzung der Schweiz als tertium gaudens des Imperialismus. ${ }^{78}$ Die Position einer Schweiz, welche die imperialen Mächte unterstützt und dabei ihre eigenen Interessen vertritt, konnte durchaus auch dem eigenen Selbstverständ-

75 | Siehe etwa zur Aktivität von Schweizer Missionaren und Missionarinnen und deren Rolle innerhalb der Anthropologie Harries, Butterflies and Barbarians, 2007.

76 | Lang, "Schweizer Initiativen zur Wiedergutmachung der Sklaverei“. Ähnliches hatte drei Jahre zuvor auch die Schweizer Nationalrätin Pia Hollenstein vergeblich verlangt: "Die entscheidende Frage ist nicht, ob die Schweiz eine Kolonialmacht war, sondern ob sie am gesamteuropäischen ökonomischen System der Ausbeutung Afrikas und der Neuen Welt durch Sklavenhandel, Plantagenwirtschaft und Kolonialwarenhandel partizipiert und davon profitiert hat."So die Stellungnahme von Pia Hollenstein auf die Antwort des Bundesrates vom 16.06.2003 in: Hollenstein, "Interpellation Hollenstein Pia".

77 | Vgl. Thürmer-Rohr, Vagabundinnen.

78 | Vgl. Witschi, Schweizer. Zu einem anderen Schluss kommt Andrea Franc in ihrer Untersuchung der Aktivitäten der Basler Handelsgesellschaft (BHG) an der damaligen Goldküste, in der sie feststellt, dass sich eine sekundärimperialistische Protektion durch die Briten nur ein einziges Mal, nämlich während der Aufstände von 1948, feststellen lässt (Franc, Wie die Schweiz, S. 218). Falls von einer "Trittbrettfahrerin" der kolonialen Situation die Rede sein könne, so Franc, würde das nur die BHG betreffen und nicht den Schweizer Staat, der sich in der Goldküste nicht engagiert habe (ebd., S. 225). 
nis entsprechen. So meinte im Juli 1847, wenige Monate vor dem Ausbruch des Sonderbundskriegs, Ulrich Ochsenbein, damaliger Präsident der Tagsatzung und späterer Bundesrat: »Auf dem ganzen Erdenrund, soweit die Beharrlichkeit des kühnen Briten festen Fuss gefasst, findet Ihr den Schweizer als treuen Begleiter an seiner Seite, einen Absatz zu suchen für die Produkte der Kunst und des Fleisses seines Vaterlandes.«79

Lorenz Stucki geht einen Schritt weiter und bezeichnet die Schweiz 1968 selbst als Imperium, wenn auch als »heimliches Imperium«, über dem »keine Schweizer Fahnen « ${ }^{8 \circ}$ wehen würden. Roland Ruffieux spricht von einem »Bank- und Börsenimperialismus «, den die Schweiz im 19. Jahrhundert im Windschatten der Kolonialmächte entwickelt habe, und attestiert der Schweizer Beteiligung an internationalen kapitalistischen Unternehmungen den »Charakter eines verdeckten Kolonialismus « ${ }^{81}$. Hans Fässler verwendet in Anlehnung an Herbert Lüthys Analyse der Beteiligung von Schweizer Unternehmungen am transatlantischen Sklavenhandel den Begriff der »Teilzeit-Kolonialmacht « ${ }^{82}$. Auch Thomas David und Bouda Etemad zeichnen die Umrisse eines »Schweizer Imperialismus « nach. ${ }^{83}$ Wie die meisten anderen Autorinnen und Autoren betonen sie dessen »Mehrdeutigkeit ${ }^{84}$. Neutralität und Humanität stehen demnach nicht grundsätzlich in einem Widerspruch zum imperialen Gebahren der Schweiz - auch wenn dies punktuell der Fall sein kann -, sondern vielmehr in einem konstitutiven Verhältnis zu ihm: Für die Schweiz, so David und Etemad, »fielen aus den humanitären Bestrebungen immaterielle Zinsen ab: moralisches Prestige und internationale Reputation « ${ }^{85}$. Auch Jean Ziegler, der 1976 die globalen Verflechtungen unter einer US-amerikanischen Hegemonie untersucht, geht von einem »sekundären schweizerischen Imperialismus« aus, der »unerlässliche, genau umrissene Aufgaben innerhalb ein und desselben weltbeherrschenden Systems « ${ }^{86}$ übernehme.

Der Imperialismusbegriff ist in einer marxistischen Denktradition zu verorten. Nach Rosa Luxemburg war der Imperialismus dem Kapitalismus inhärent und markierte zugleich dessen letztes Stadium: Der Kapitalismus müsse notwendigerweise immer weitere Teile der Welt erschließen, dadurch käme er seinem Zusammenbruch stets näher. ${ }^{87}$ Jürgen Osterhammel macht darauf aufmerksam, dass der Imperialismusbegriff »in den frühen 185 oer Jahren in der britischen und

79 | Ochsenbein, "Präsidialvortrag", S. $216 f$.

80 | Stucki, Das heimliche Imperium, S. 10.

81 | Ruffieux, "Schweiz des Freisinns", S. 712.

82 | Fässler, Reise in Schwarz-Weiss, S. 288.

83 | David/Etemad 1998, "Gibt es einen schweizerischen Imperialismus?"

84 | Ebd., S. 25.

$85 \mid$ Ebd., S. 24.

86 | Ziegler, Schweizer Imperium, S. 51.

87 | Luxemburg, Die Akkumulation. Dazu auch Groh, "Imperialismus". In den Geschichtlichen Grundbegriffen findet sich kein Eintrag zu "Kolonialismus". 
deutschen Publizistik zur polemischen Bezeichnung jener von ihren Gegnern als usurpatorisch betrachteten Herrschaftsform « ${ }^{88}$ verwendet wurde. Nicht immer wird im heutigen Sprachgebrauch allerdings die Geschichtlichkeit des Begriffes reflektiert. Osterhammels Einschätzung, die neuerlich zu beobachtende Ausweitung der Begriffe Imperium und Imperialismus führe zwar zu einem Verlust an terminologischer Schärfe, ermögliche es aber, bisher verdeckte Analogien und Zusammenhänge zu sehen ${ }^{89}$, gilt auch für den Kolonialismusbegriff.

Wenn wir in dieser Publikation die Bezeichnung einer »postkolonialen Schweiz $«^{9} \mathrm{zu}$ konturieren versuchen, dann steht diese sowohl in einer Kontinuität mit den erwähnten Erforschungen des »Schweizer Imperialismus« als auch für einen spezifisch kulturwissenschaftlichen Zugriff, der unseren Ansatz von jenen unterscheidet. ${ }^{11}$ Die begriffliche Differenzierung zwischen »Imperialismus « und »Kolonialismus« ist allerdings nicht stabil, sie änderte sich mehrmals im Laufe der Zeit. Im Historischen Lexikon der Schweiz wird beim Begriff Imperialismus auf den Eintrag unter Kolonialismus verwiesen; da wiederum wird Imperialismus mit dem Höhepunkt der Kolonialherrschaft vor dem Ersten Weltkrieg in Verbindung gebracht. ${ }^{92}$ Eine etwas anders gelagerte Begriffsunterscheidung finden wir bei Ania Loomba: »Thus the imperial country is the >metropole< from which power flows, and the colony or neo-colony is the place which it penetrates and controls.«93 Die Loomba'sche Definition beschreibt die heute vorherrschende Begriffsverwendung in den Imperialismus- und Kolonialismusstudien treffend. Doch ihre darauffolgende Charakterisierung - »[i]mperialism can function without formal colonies (as in United States imperialism today) but colonialism cannot «94 - entspricht gerade nicht unserem Sprachgebrauch. Schließlich bringt der Imperialismusbegriff, der auf den lateinischen Terminus imperium zurückgeht, was Befehl, Herrschaft oder Staatsgewalt bezeichnet, die Gewaltausübung in der Begriffsbildung expliziter zum Ausdruck; gleichzeitig geraten vielleicht weniger deutlich sichtbare Gewalt-

88 | Osterhammel, "Imperialismus", S. 536.

89 | Vgl. ebd., S. 537.

90 | Bernhard Schär schlägt 2007 vor, eine "postkoloniale Geschichte der Schweiz" zu verfassen (Schär, "Mariella Mehr"). Ein erster Versuch, den Begriff der postkolonialen Schweiz zu umreißen, findet sich in Purtschert, "Postkoloniale Diskurse" und Purtschert, "Heute bedankt sich Naresh Khan “. Zur postkolonialen Schweiz vgl. zudem Salgado, "Chewing the Borders".

91 | Es bleibt dabei ein Forschungsdesiderat, das Kulturelle und das Ökonomische nicht als Oppositionen, sondern in ihrer gegenseitigen Konsitutierung zu sehen.

92 | Von Albertini/Wirz, "Kolonialismus".

93 | Loomba, Colonialism, S. 12.

94 | Ebd. Diese Begriffsverwendung macht zudem die postkoloniale Situation der Native Americans in den USA unsichtbar. 
ausübungen schneller aus dem Blick. ${ }^{95}$ Kolonialismus wiederum bezeichnet nach Jürgen Osterhammel in einem engeren Sinne den »Prozess « der Landnahme, ${ }^{96}$ allgemeiner kann er auch ein Herrschaftsverhältnis benennen. Wie der Begriff der Kultur, geht er auf das lateinische Verb colere zurück, das unter anderem mit bebauen, bestellen und bewohnen übersetzt wird. Für unser Erkenntnisinteresse ist diese kulturelle Konnotation relevant. Allerdings kann auf die Etymologie auch in euphemistischer Absicht zurückgegriffen werden, bedeutet doch colere auch ausbilden oder Sorge tragen.

Patrick Minder hat kürzlich die Bezeichnung der Suisse coloniale in die aktuelle Diskussion eingeführt. ${ }^{97}$ Seine Studie, die das koloniale Imaginäre der Schweiz untersucht, konzentriert sich auf die Zeit zwischen 1880-1939 und macht in ihrer Materialvielfalt deutlich, wie angezeigt die Rede von einer »kolonialen Schweiz« ist. ${ }^{8} \mathrm{Im}$ Unterschied zu Minders Zugang, welcher die (Dis-)Kontinuitäten zur Gegenwart nicht explizit zum Thema macht, wollen wir gerade diese Aspekte mit thematisieren. Der Begriff der postkolonialen Schweiz umfasst dabei verschiedene Facetten. Historisch betrachtet fokussiert er auf das nachkoloniale Zeitalter, macht aber zugleich deutlich, dass dieses sich nicht ohne Bezug auf den Kolonialismus deuten lässt. Eine postkoloniale Perspektive erlaubt es demnach, die Gleichzeitigkeit von antikolonialen Kämpfen und dem Weiterwirken von kolonialen Mustern in den Blick zu nehmen. ${ }^{99}$ Der Begriff transportiert weiter eine Fülle von theoretischen, inhaltlichen und methodischen Aspekten der postcolonial studies und bringt diese mit der Schweiz als Forschungsgegenstand in Verbindung. Diese kulturwissenschaftliche Konnotation des Postkolonialen ermöglicht es, über wirtschaftliche und politische Verflechtungen hinaus Bezüge zum Schweizer Alltag, zur Wissenschaft und zu Populärkulturen herzustellen, indem der Repräsentation, der Imagination, dem Diskursiven und Visuellen besondere Aufmerksamkeit geschenkt wird. ${ }^{100}$ Welche Rolle kommt etwa den kolonialen Vorstellungen und Bildern zu,

95 | Bereits der Begriff der strukturellen Gewalt wollte indes für unsichtbare Gewaltdimensionen sensibilisieren: "Die Unterscheidung personale/strukturelle Gewalt geht insofern mit der Unterscheidung sichtbar/unsichtbar einher. Während im ersten Fall der Verursacher des Schadens klar in Erscheinung tritt, bleibt er im Falle der strukturellen Gewalt unsichtbar und ist schwer auszumachen." Schroer, "Gewalt ohne Gesicht", S. 156.

96 | Osterhammel, Kolonialismus, S. 8.

97 | Minder, La Suisse coloniale.

98 | Zur aktuellen Diskussion über die "koloniale Schweiz" vgl. auch die gleichnamige Studie von Andreas Zangger (Zangger, Koloniale Schweiz).

99 | Mackenthun, "E Pluribus Unum ", S. 375.

100 | Ein erster Versuch, derartige Fragen zu beantworten, wurde auf einer Konferenz an der Universität Basel im Jahre 2003 unternommen: Imperial Culture in Countries Without CoIonies: Africa and Switzerland, Universität Basel, 23.-25. Oktober 2003; siehe dazu http:// pages.unibas.ch/afrika/nocolonies, 21.04.2011. Wegweisend war auch die 2005/06 durchgeführte Projektreihe der Shedhalle Zürich unter dem Titel Kolonialismus ohne Ko- 
die in der Schweiz omnipräsent waren und die - in abgeänderter und herkömmlicher Gestalt - weiterhin zirkulieren? ${ }^{101}$ Auch wenn die Sphären des Populären, Wissenschaftlichen, Sozialen und Politischen zuweilen eigene Logiken ausbilden, die sich nicht einfach ineinander übersetzen lassen, gehen wir davon aus, dass die Untersuchung der Relationen, Zusammenhänge und wechselseitigen Bezüge zwischen diesen unterschiedlichen Bereichen von großem Gewinn sein kann. Dabei leistet das Konzept der postkolonialen Schweiz eine wichtige Transferfunktion: Denn die Arbeit, welche im Rahmen der postcolonial studies in den vergangenen Jahrzehnten geleistet wurde, war auch eine Arbeit am Begriff. Die Konturen einer »postkolonialen Schweiz« zu umreißen bedeutet derart, die Schweiz mit der Theoriemaschine des Postkolonialismus zu verkoppeln. Es bedeutet, Machtverhältnisse zu artikulieren, das Transnationale in den Blick zu nehmen, die Inter- und Transdisziplinärität stark zu machen, Vorstellungen von Nation, »Rasse« oder Kultur zu dekonstruieren oder die intersektionalen Verbindungen zwischen Kolonialismus, Sexismus, Homophobie, Klassenkonflikten und anderen strukturellen Machtverhältnissen zu erörtern. Weiter macht die »postkoloniale Schweiz« explizit, dass es Verbindungen zum Kolonialismus gibt, ohne bereits eine Vorentscheidung darüber zu fällen, ob es um Komplizenschaft oder andere Formen der Partizipation geht. Der Begriff der Komplizenschaft ist situativ hilfreich, um die Involviertheit von Akteurinnen und Akteuren zu beschreiben. Weil er allerdings ein intentionales Handeln suggeriert, eignet er sich nur bedingt, die strukturellen Dimensionen einer postkolonialen Schweiz zu erfassen. Denn es ist gerade ein Kennzeichen des strukturellen Rassismus, dass er von den Menschen, die ihn reproduzieren, oftmals nicht als solcher erkannt, sondern beispielsweise als Bestandteil der »Schweizer Alltagskultur« erachtet wird.

\section{TransNational BEDEUTET NICHT POSTNATIONAL}

Eine kritische Frage bleibt unvermeidbar, wenn von der postkolonialen Schweiz die Rede ist: Ist der Begriff mit seiner Fokussierung auf die Schweiz nicht kontraproduktiv und anachronistisch? Was bringt ein national fokussierter Zugang, wenn der Postkolonialismus gerade das Transnationale betonen und die Vorstellung einer uneingeschränkten nationalen Souveränität als eurozentrischen My-

Ionien? Beziehungen zwischen Tourismus, Neokolonismus und Migration; siehe hierzu http://archiv.shedhalle.ch/dt/archiv/2006/programm/thematische_reihe/konzept/ index.shtml, 21.04.2011, die beiden Workshops zur postkolonialen Schweiz, die am 7. und 8. November 2008 an der Universität Basel und am 7. November 2009 an der ETH Zürich stattgefunden haben, sowie die Gründung der an den Universitäten Genf und Lausanne angesiedelten Forschungsgruppe POST IT ("Penser la différence postcoloniale et racial») im Jahre 2008.

101 | Vgl dazu dos Santos Pinto/Lienhard/Purtschert, "Die postkoloniale Schweiz". 
thos dekonstruieren will? Wäre es nicht angebracht, auf einen nationalen Bezug zu verzichten und die zurzeit noch weitgehend nationalstaatlich geführten Auseinandersetzungen in europäische Debatten münden zu lassen? ${ }^{102}$ Wenn die Kolonialismusforschung, die sich auf einzelne nationale Räume beschränkt, zu Recht aus einer transnationalen und postkolonialen Perspektive kritisiert werden kann: Wie lässt sich ein Projekt wie das unsere dann noch legitimieren? ${ }^{103}$

Die Frage nach einer postkolonialen Schweiz ergibt insofern Sinn, als die bestehende Spannung zwischen Nationalität und Transnationalität keineswegs die Auflösung einer Seite bedeutet, sondern als komplexes Wechselspiel verstanden werden kann. Unsere Analyse geht nicht von einem Verständnis der Schweiz aus, welche als fixe Größe in das koloniale Geschehen eingegriffen hat. Was »die Schweiz« ist, wie schweizerische Akteure entstehen, agieren und wieder verschwinden, kann vielmehr nur aufgrund der transnationalen Verbindungen ausgemacht werden, in die sie eingebunden ist, und - mehr noch - aus denen sie konstituiert wird. Umgekehrt kann der Bereich des Transnationalen aber auch nicht beschrieben werden, ohne die Interventionen, Verknüpfungen, Abgrenzungen, Konfrontationen und Zusammenschlüsse zu verstehen, welche sich im Namen des Nationalen ereignen. ${ }^{104}$ Mit anderen Worten: Auch wenn Nationalstaaten auf Konstruktionsprozesse zurückgehen, beruhen sie auf äußerst wirkmächtigen »imagined communities ${ }^{105}$ und nationalstaatlich festgelegten und kontrollierten Praktiken wie Grenzregelungen, Einreise- und Ausweisungsverfahren oder behördlichen Prozeduren, die an nationalstaatliche Unterscheidungskriterien gebunden sind.

Das Beharrungsvermögen des Nationalen wird auch in Bezug auf institutionelle Akteure festgestellt. So halten Andreas Eckert und Shalini Randeria in Abgrenzung zur behaupteten Erosion staatlicher Macht fest, dass »der Staat und seine rechtlichen Praktiken weiterhin grundlegend für das Funktionieren des internationalen Rechts und internationaler Institutionen ${ }^{106}$ seien. Sie schlagen vor, »das Nationale und das Internationale eher [als] miteinander verzahnt, als [...] sich diametral gegenüberstehen[d]« zu verstehen. ${ }^{107}$ Der nationale Fokus, den die postkoloniale Schweiz auf den Plan ruft, ist somit eine Frage der Perspektive: Ausgangspunkt ist nicht ein autarker Nationalstaat, sondern die Beschreibung transnationaler Prozesse aus der Sicht eines auf spezifische Weise in das koloniale Regime eingebundenen Ortes. So ist beispielsweise die Amnesie in Bezug auf kolo-

102 | Vgl. dazu Eckert, "Der Kolonialismus".

103 | Castro Varela/Dhawan, "Mission Impossible", S. 309.

104 | Vgl. etwa Clavin, "Defining Transnationalism«; Patel, "Transnationale Geschichte»; Tyrell, Transnational Nation, S. 3ff.

105 | Anderson, Imagined Communities.

106 | Eckert/Randeria, Vom Imperialismus zum Empire, S. 16.

107 | Ebd. Siehe dazu allgemein Iriye, "Internationalization" und Patel, "Transnationale Geschichte". 
niale Beziehungen ein europäisches Phänomen. Dennoch lassen sich sowohl transnationale wie auch nationale Züge dieser »Kolonialismusvergessenheiten« ausmachen. Während ehemalige Kolonialmächte dazu neigen, die koloniale Vergangenheit zu verdrängen, herunterzuspielen oder ihre »positiven Aspekte « zu betonen, wird in der Schweiz (wie auch in anderen Ländern, welche keine formalen Kolonialmächte waren) sowohl von Regierungsseite als auch in öffentlichen Diskussionen zumeist die Ansicht vertreten, die Schweiz habe mit dem Kolonialismus und dem transatlantischen Sklavenhandel nichts zu tun. ${ }^{108}$

Was nun kann der Begriff einer postkolonialen Schweiz erklären, was kann er in Bewegung bringen, welche Erkenntnisse ermöglicht er? Anhand von sechs zentralen Konzepten der postkolonialen Theorie - entanglement, Spektakel des Exotischen, Warenrassismus, othering, koloniale Wissensproduktion und Widerstand - umreißen wir im Folgenden einige Forschungsfelder und skizzieren dabei mögliche Umrisse einer postkolonialen Schweiz.

\section{SCHWEIZER ENTANGLEMENTS}

Der Blick auf die Aufarbeitung der kolonialen Vergangenheit in Frankreich, Italien, Deutschland und anderen europäischen Ländern ist für die Schweiz nicht nur interessant, weil er einen Vergleich mit den Nachbarstaaten und innerhalb Europas ermöglicht. Es zeigt sich vielmehr auch, dass die Forschungstendenzen in eine Richtung gehen, die für die Schweiz in Ansätzen anschlussfähig ist: Zum einen werden vermehrt »Alltagsrassismen « in den Blick genommen, die darauf verweisen, dass rassistische Bilder über Nationengrenzen hinweg zirkulier(t)en. ${ }^{109}$ Zum anderen hat die These, wonach sich der Kolonialismus nicht parallel in unterschiedlichen Ländern ereignet hat, sondern ganz wesentlich ein länderübergreifendes Unternehmen war, Folgen für die Schweiz. Die Beteiligung der Schweiz an der europäischen Kolonialgeschichte anhand von transnationalen Verflechtungen, als entanglement zu schreiben bedeutet folglich, die Geschichte des Kolonialismus als ein gemeinsames europäisches Erbe zu verstehen. ${ }^{110}$ Anhand eines Beispiels soll diese Verwicklung der Schweiz aufgezeigt werden.

Wenig bekannt ist, dass sich Henry Dunant, bevor er das Rote Kreuz gründete, als Kolonialunternehmer in Algerien versuchte. 1852 initiierte eine Genfer Gesell-

108 | Vgl. dazu die von Jean-Daniel Vigny im Vorfeld der Weltkonferenz gegen Rassismus in Durban 2001 getroffene Aussage, die zu einem symbolträchtigen Ausdruck dieser Haltung wurde. Vigny wird zitiert in Egli, "Weder Entschuldigung noch Entschädigung", S. 16. Zur Geschichte und Bedeutung seiner Aussage vgl. Purtschert, "Chewing on Post_colonial Switzerland, Part I, II und III".

109 | Dazu auch die hier publizierten Arbeiten von Rohit Jain und Christine Bischoff.

110 | Paradigmatisch für den Zugang des entaglement ist Conrad/Randeria, "Geteilte Geschichten". 
schaft eine Kolonie im heute algerischen Sétif. Es war Dunant, der im Auftrag der Compagnie genevoise des colonies suisses de Sétif eine dazugehörende Finanzgesellschaft gründete, die mit dem angebauten Getreide handelte, Mühlen betrieb und Vieh züchtete. ${ }^{111}$ Zudem wollte Dunant in Algerien eine eigene Mühle in Betrieb nehmen. ${ }^{112}$ Nach Solferino war er in der Hoffnung gereist, den französischen Kaiser für seine kolonialen Geschäfte zu gewinnen. ${ }^{113}$ In Algerien lebten in den 188 oer Jahren über 3.000 Schweizerinnen und Schweizer. Hinter dieser Genfer Koloniegründung stand die Überzeugung, dass das koloniale Algerien Chancen biete, die zeitig ergriffen werden mussten: »La Compagnie genevoise fut crée sans plan de développement préétabli sur la conviction que l'Algérie coloniale offrait des opportunités qu'il fallait saisir précocement. «114 Zudem konnten Gemeinden durch Koloniegründungen Fürsorgekosten sparen. Allerdings war das Unternehmen in Algerien nicht nur von Erfolg gekrönt. Die Schweizerinnen und Schweizer konnten auch aufgrund höherer Löhne nicht mit einheimischen Bauern und Bäuerinnen konkurrieren und die dortige geringe Bevölkerungsdichte erschwerte den Zugriff auf ausbeutbare Arbeitskräfte. ${ }^{115}$

Sétif und seine benachbarten Orte wurden am 8. Mai 1945 Schauplatz eines Massakers gegen die algerische Bevölkerung. Dieses Datum gilt heute als Ausgangspunkt für den 1954 beginnenden Algerienkrieg. Es ist wahrscheinlich, dass auch Schweizer Söldner an diesem Massaker beteiligt waren, denn diese machten etwa sechs bis acht Prozent der französischen Fremdenlegion aus. ${ }^{116}$ Später, während des Algerienkrieges, konnten die Anhänger der französischen Kolonie auf die aktive Sympathie der ansässigen Schweizer Bevölkerung zählen, zugleich stand die Schweiz aber sowohl algerischen Unabhängigkeitskämpfern wie auch Deserteuren als Aufenthalts- und Durchgangsland offen. ${ }^{117}$ In der Schweiz erschienen zudem Schriften, die den französischen Kolonialismus in Algerien kritisierten, so beispielsweise diejenigen des ehemaligen Söldners Franz Rispy, der im Ruf stand, mit dem algerischen Front de Libération Nationale zusammenzuarbeiten. Nach dem Algerienkrieg konnte sich die Schweiz als diplomatische Vermittlerin ohne koloniale Vergangenheit darstellen, was für ihre eigene, nach dem Zweiten Weltkrieg isolierte Position im internationalen Staatengefüge vorteilhaft war. ${ }^{118} \mathrm{Nach}$

111 | Nach zahlreichen Misserfolgen wurde Dunant allerdings als Leiter abgesetzt. Perrenoud, "Algerien ", S. 183.

112 | Steiner, Henry Dunant, S. 89.

113 | Wirz, "Humanitäre Schweiz", S. 96.

114 | Lützelschwab, Compagnie, S. 354.

115 | Ebd., S. 357.

116 | Die französische Fremdenlegion gelangte vor allem in kolonialen Gebieten zum Einsatz, als erstes in den dreißiger und vierziger Jahren des 19. Jahrhunderts bei der Eroberung Algeriens. Siehe dazu den Artikel von Christian Koller in diesem Band.

117 | Perrenoud, "Algerien", S. 182.

118 | Perrenoud, "Aperçu«, S. 342. 
der Unabhängigkeit Algeriens verlangten die in Algerien lebenden Schweizerinnen und Schweizer aufgrund der durchgeführten Verstaatlichung Entschädigungen, die Algerien jedoch verweigerte. ${ }^{119}$ Dieses Beispiel zeigt spezifisch helvetische Züge: Die »Schweiz« erscheint dabei als Akteurin, die sich je nach Situation auf »beiden Seiten« bewegen kann. Dass eine solche Beteiligung am europäischen Kolonialismus für die Interpretation der Geschichte und Gegenwart der Schweiz Folgen hatte, wird nicht erst heute erkannt. So wurde die heute wenig bekannte Vorgeschichte von Henry Dunant bereits vor 30 Jahren in einer Monographie von Jacques Pous aufgearbeitet. Der Autor kam dabei zu folgendem Schluss:

"La participation d'Henry Dunant au grand mouvement d'expansion coloniale qui commence alors à ébranler l'Europe, pose en réalité la question de l'existence et de la spécificité d'un colonialisme helvétique. La reconnaissance de l'impérialisme d'un pays sans colonie ne va pas en général de soi, surtout que, la plupart du temps, le monde feutré et cosmopolite des affaires n'a pas pour objectif de rendre transparents les mécanismes de la domination. "120

Wie Pous darlegt, besteht und bestand allerdings wenig Interesse, solche vergangenen und gegenwärtigen Verflechtungen sichtbar zu machen. Das Algerien-Beispiel verdeutlicht, dass Schweizer Firmen, Akteure, Institutionen und auch der Schweizer Nationalstaat auf vielfache, teilweise auch widersprüchliche Weise in die Unternehmungen der kolonialen Regimes verflochten waren.

Ein anderes Beispiel für eine »verschränkte Geschichte« ist die Internierung nordafrikanischer Soldaten im Dienste der französischen Kolonialmacht, sogenannter Spahis, in der Schweiz. So stammte ein Teil der fast 90.000 internierten Soldaten der Bourbaki-Armee, die 1871 die Schweizer Grenze übertreten haben, aus Nordafrika. ${ }^{121}$ Auch als im Juni 1940 das Gros des 45. Französischen Armeekorps in die Schweiz übertrat, befanden sich 1100 algerische Soldaten, sogenannte Spahis, darunter. ${ }^{122}$ In einer kürzlich veröffentlichten mikrohistorisch angelegten Studie zeigt Manuel Menrath, welche Folgen die Anwesenheit der nordafrikanischen Soldaten im luzernerischen Dorf Triengen hatten: Der Pfarrer ist dabei genauso ein Akteur wie die Dorfkinder, junge Männer und Frauen, der gemeinnützige Frauenverein, die Dorfbehörden, lokale Medien, Schweizer Militärvertreter und die Spahis selbst. Die Reaktionen bewegten sich zwischen verordneter Abstandnahme und einer Vielfalt von Kontakten, die sich zwischen der lokalen Bevölkerung und den internierten Männern ergaben. ${ }^{123}$

\footnotetext{
119 | Perrenoud, "Algerien".

120 | Pous, Henry Dunant, S. 177.

121 | Menrath, Exotische Soldaten, S. 55.

122 | Ebd., S. 68.
}

123 | Der beunruhigte Gemeinderat ließ etwa im Trienger Anzeiger eine Anweisung veröffentlichen, in der es heißt: "Die Bevölkerung wird dringend ersucht, im Verkehr mit den 


\section{Exotismus als Schweizer Spektakel}

Eine andere Auseinandersetzung mit der postkolonialen Schweiz wird möglich, wenn die Zelebration und Popularisierung von stereotypen Fremdbildern betrachtet wird. Ein historisches Beispiel dafür sind die seit Mitte des 19. Jahrhunderts beliebten Völkerschauen, die als Volksbelustigung galten und oft von Ort zu Ort zogen. Manche wurden von lokalen Veranstaltern organisiert, oftmals fanden sich Schweizer Städte und Dörfer aber auch auf dem Tourneeplan von international agierenden Schaubuden. So wurde die Nachricht vom Eintreffen des Extrazugs mit der »Singhalesen-Show « des deutschen Carl Hagenbeck am 1. August 1885 in Zürich begeistert aufgenommen. Bereits am ersten Wochenende wurden über 10.000 Eintrittskarten verkauft und die Neue Zürcher Zeitung riet den Leserinnen und Lesern nach einer Woche: »Es genügt nicht, die Singhalesen nur ein einziges Mal zu besuchen. Die Tänze, welche sie aufführen, der ganze ungewöhnliche Aufputz, alles muthet uns fremdartig an. Aber bei öfterem Besuch beginnt die Phantasie diese fremden Bilder zu verarbeiten und man fühlt sich in einen Palmenhain versetzt. $\ll^{124}$ Zwischen der Ausstellung eines westafrikanischen Mannes um 1835 und der afrikanischen Tier- und Völkerschau, die der Zirkus Knie 1960 (!) auf der Sechseläutewiese veranstaltet hat, macht Rea Brändle in Zürich über 60 Völkerschauen aus. ${ }^{125}$

Timothy Mitchell hat gezeigt, dass diese »Kultur des Spektakels« Teil einer spezifisch kolonialen Repräsentationspraxis ist, welche die Welt als Ausstellung versteht:

"Während des gesamten 19. Jahrhunderts sahen sich nichteuropäische Besucher zur Schau gestellt oder in das Objekt intensiver europäischer Neugier verwandelt. Die Erniedrigung, die sie oft erlitten, war, ob beabsichtigt oder nicht, anscheinend unvermeidlich und war für dieses Spektakel ebenso notwendig wie die an Gestellen befestigten Fassaden oder die Scharen neugieriger Zuschauer. ${ }^{126}$

Ein Effekt dieser Praxis bestand darin, dass die westliche Wahrnehmung von anderen Kulturen wesentlich durch eine Logik bestimmt war, die auf die eigene, das heißt eurozentrische Repräsentation zurückgeht. Dies zeigt sich auch bei den europäischen Reisenden, welche die Ausstellungen verließen, um das reale Andere aufzusuchen ${ }^{127}$ : Oftmals rückten sie ihre Erlebnisse (unter großem epistemischen

internierten Truppen den notwendigen Abstand zu wahren. Junge Mädchen und Frauen werden darauf aufmerksam gemacht, dass besonders Soldaten der farbigen Truppe in gesundheitlicher und sittlicher Beziehung nicht einwandfrei sind "(ebd., S. 119).

124 | Brändle, Wildfremd, hautnah, S. 49.

125 | Vgl. Ebd., S. 160-166.

126 | Mitchell, "Welt als Ausstellung", S. 150.

127 | Siehe dazu auch den Beitrag von Gaby Fierz in diesem Band. 
Aufwand) so zurecht, dass sie den mitgebrachten Bildern entsprachen. Bezeichnend für die koloniale Logik des Ordnens und Darstellens war auch die verbreitete Zurschaustellung von exotisierten Menschen in Schweizer Zoos. ${ }^{128}$

Die koloniale Tradition einer »Kultur des Spektakels« kann in der Schweiz bis in die Gegenwart hinein verfolgt werden. So bietet beispielsweise die Fasnacht reichlich Stoff für eine postkoloniale Analyse: Noch heute verkleiden sich zahlreiche Schweizerinnen und Schweizer als Indianerhäuptlinge mit Federschmuck, Afrikanerinnen im Baströcklein oder Chinesen mit langem Zopf. Die Präsenz solcher kolonialen Spektakel im Schweizer Alltag geht einher mit dem kargen Wissen über den Kolonialismus und den außereuropäischen Raum, das den Schweizer Alltag kennzeichnet. Das führt, wie Cintia Meier-Mesquita beschreibt, etwa zu Zweifeln an der nationalen Zugehörigkeit von Menschen, die als nicht weiß taxiert werden. Ein Personalchef habe das Vorzeigen ihres portugiesischen Passes mit der Aussage quittiert:

"Portugiesen sind weiss, Sie aber sind schwarz. [...] Dies wirkte auf mich sehr befremdend, weil ich konstatieren musste, dass erstens Dunkelhäutige - auch wenn sie an der Universität tätig sind - nicht als glaubwürdig gelten, und dass zweitens hierzulande [in der Schweiz] die Kolonialgeschichte Europas weitgehend unbekannt ist. «129

\section{SCHWEIZER WARENRASSISMUS}

Ein weiteres Beispiel dafür, wie der Rassismus in den Schweizer Alltag diffundiert und dort so sprichwörtlich »alltäglich« geworden ist, dass er kaum als solcher wahrgenommen wird, lässt sich in dem Bereich ausmachen, den Anne McClintock als »Warenrassismus « (commodity racism) bezeichnet. ${ }^{130}$ McClintock geht davon aus, dass die Bedeutung eines im Rahmen der wissenschaftlichen Forschung entwickelten Rassismus auf eine kleine, wenn auch bedeutsame Elite beschränkt blieb. Mit der Entwicklung des Kapitalismus hingegen, der zur zunehmenden Zirkulation von Gütern und zur Adressierung breiterer Gesellschaftsschichten in Westeuropa als Konsumentinnen und Konsumenten führte, entstand eine neue und ungeheuer wirkmächtige Form des Rassismus, die sich vor allem über Konsumgüter verbreitete und mit deren Erwerb und Verzehr unweigerlich verknüpft war. $^{131}$

128 | Vgl. die Untersuchung von Balthasar Staehelin zum Zoo Basel: "Zwischen 1879 und 1935 wurden dem Basler Zoopublikum einundzwanzig Schauen mit meist aussereuropäischen Menschen vorgeführt."Staehlin, Völkerschauen, S. 11. Vgl. dazu auch Minder, "La construction du colonisé".

129 | Meier-Mesquita, "Soziale Bedeutung", S. 9.

130 | Vgl. McClintock, Imperial Leather, S. 31-36, insbesondere S. 33.

131 | Ebd., S. 209. 
Abbildung 1: Werbung für » Weisse Waren«, 1933

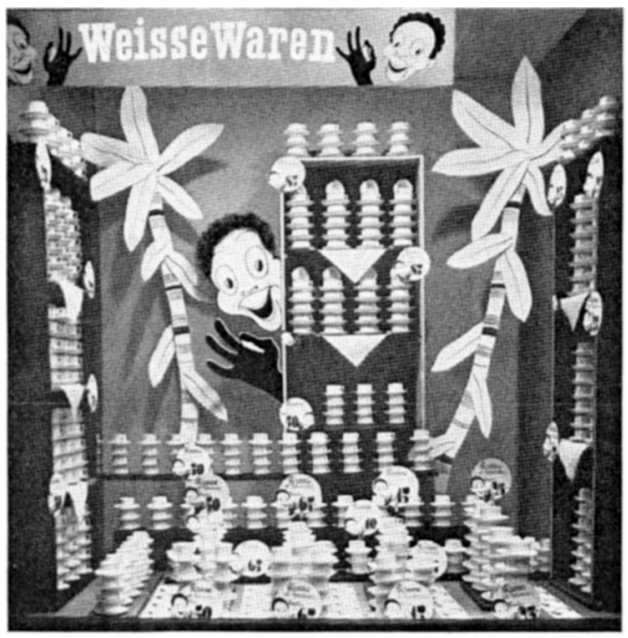

Ein Schaufenster der Filiale Basel

Ein Schweizer Beispiel dafür ist die Lancierung einer Werbefigur des Schweizer Warenhauses Globus. Neben »Globi«, dem blauen Papagei in der rot-schwarz karierten Hose, der die Kinder auf seinen Geschichten nicht selten an exotische und von kolonialen Bildern nur so strotzende Orte mitnahm, wurde in den 193oer Jahren die Figur des »weißen Negers « aus Afrika eingeführt. ${ }^{132}$ Er sollte erst dabei behilflich sein, weiße Wäsche, sogenannte Weißwaren, an die Schweizer Hausfrau $\mathrm{zu}$ bringen, und wurde später in einigen Geschichten zu einem Kameraden von Globi. In einem Globus-Inserat von 1935 werden die Kinder aufgerufen, ihre Mütter zum Kauf von Weißwaren aufzufordern, denn Globi habe »seinen Freund den weißen Neger - damit beauftragt, in allen Rayons schöne und billige weiße Waren zu besorgen « ${ }^{133}$. Das Inserat ist umrahmt von elf Bildern, auf denen der »weiße Neger« verschiedenen Kindern Hüte, Schuhe, Strümpfe, Kleider und andere Waren anbietet. Zumeist ist er in der Haltung eines Dieners dargestellt. Auf einigen Bildern weist er auffallend feminine Züge auf. Ins Auge sticht auch, dass die Arme und Beine des »weißen Negers« schwarz koloriert sind. Damit wird die Assoziation geweckt, der »weiße Neger« sei ein Schwarzer mit weiß geschrubbtem Gesicht. Diese sogenannte Mohrenwäsche hatte in der Alltagskultur des ausgehenden 19. Jahrhunderts einen festen Platz, wie Nana Badenberg gezeigt hat. ${ }^{134}$ Verbreitet waren Geschichten von »Mohren«, die dank ihres guten Herzens weiß, oder, umgekehrt, von Kindern, die aufgrund von übermäßigem Schokoladenkon-

132 | Zur Figur von Globi siehe den Artikel von Patricia Purtschert in diesem Band.

133 | N. N., "Weisse Waren", o. S.

134 | Badenberg, "Die Bildkarriere". 
sum schwarz wurden. In suggestiver Weise verbanden sich in diesem kulturell verfestigten Stereotyp hygienische und moralische Denkfiguren, wobei dabei auch Assoziationen von hell und dunkel, Tag und Nacht aufgerufen wurden, die älter waren und dem Kolonialismus vorangingen. 135 »Produkte, die glänzendes Weiß versprachen, setzten auf die Werbewirksamkeit Schwarzer. ${ }^{136}$ Was schwarz ist, gilt als schmutzig; »die Redewendung >einen Mohren weiß waschen < bezeichnet das aussichtlose Unterfangen, einen offenkundig Schuldigen entlasten zu wollen « ${ }^{137}$. Das sprachliche Gegenstück dazu ist das »Anschwärzen«. Die Verbindung von weißer Wäsche, einem (von seiner schwarzen Farbe) »weiß gewaschenen « Afrikaner und der Werbung weist auf den transnationalen Kontext des »commodity racism « hin: Seife, so schreibt McClintock, wurde das vorherrschende Medium, in dem sich der neue, kommerzielle Imperialismus mit dem bürgerlichen Haushaltskult verband. ${ }^{138}$

Ein anderes Beispiel für die Zirkulation kolonialer Bilder durch den kapitalistischen Kreislauf beschreibt Roman Rossfeld. Die Vermarktung der Schweizer Schokolade, so zeigt er, wurde Ende des 19. Jahrhunderts von einer Werbung dominiert, die sich im »Spannungsfeld von Exotismus und Nationalismus « befand. ${ }^{139}$ Die

"oft stereotyp gestalteten Motive ermöglichten nicht zuletzt eine deutliche Differenzierung von (nationaler) Identität und (exotischer) Alterität. Üblicherweise wurden die Schwarzen dabei mit dem Rohstoff Kakao und nicht den verarbeiteten Produkten abgebildet, was indirekt auch auf die in der bürgerlichen Gesellschaft wichtigen Dichotomien von ımodernvormodern، respektive ,neuzeitlich-archaisch`verwies und die Schwarzen zugleich einer rückständigen Lebenswelt zuordnete ${ }^{140}$

Die Schokolade als Konsumgut transportierte somit Bilder der modernen Schweiz, die mit kolonialen Vorstellungen einer vormodernen, exotischen und als Rohstofflieferant dienenden Welt untrennbar verwoben waren.

135 | Eine solche Farbsymbolik ist nicht erst im 19. Jahrhunder entstanden, vielmehr wurden bestehende farbliche Zuweisungen im Zuge der Erfindung moderner Rassen neu konfiguriert. Vgl. dazu Husmann, Schwarz-Weiss-Symbolik.

136 | Badenberg, "Die Bildkarriere", S. 177.

137 | Ebd., S. 174.

138 | "The emergent middle class values - monogamy ('clean، sex, which has value), industrial capital ('clean، money, which has value), Christianity ('being washed in the blood of the lamb`), class control ('cleansing the great unwashed`) and the imperial civilizing mission ('washing and clothing the savage،) - could all be marvelously embodied in a single household commodity." McClintock, Imperial Leather, S. 208.

139 | Rossfeld, Schweizer Schokolade, S. 436.

140 | Ebd. Vgl. auch Franc, Wie die Schweiz. 


\section{Othering à la SUISSE}

Postkoloniale Studien weisen darauf hin, dass sich Identitätsbildungen im kolonialen und postkolonialen Zeitalter über Prozesse des othering ereignen. ${ }^{141}$ Insbesondere der Selbstentwurf als moderne Nation bedient sich einer Rhetorik der Absetzung von vormodernen und archaischen Anderen. Das gilt, wie Patrick Harries festhält, auch für die Schweiz: »In Switzerland, as elsewhere in the world, the outlines of modernity were defined against a primitive other. $\ll^{142}$ Das Verhältnis moderner Schweizerinnen und Schweizer zu diesen »internen Anderen « war komplex: Einerseits verkörperten die Alpenbewohner eine primitive Welt, in der Aberglauben vorherrschte und eine archaische Lebensweise gepflegt wurde. Andererseits arbeitete der neu entstehende Nationalismus des 19. Jahrhunderts gerade mit Bildern der Schweiz, die dieses angeblich traditionelle Leben hochhält und mit typisch schweizerischen Tugenden verbindet. Der Alpenbewohner schillerte zudem zwischen dem edlen und dem bösen Wilden: Er war der Primitive, aber auch der Inbegriff einer heilen Welt, die sich im Kontext des Kulturpessimismus mit der Nostalgie für ein ursprüngliches natürliches Leben verband. So zeigt Bernhard Schär in seiner Arbeit zur Kariesbekämpfung in der Schweiz, dass die Zähne der Alpenbevölkerung anfangs des 20. Jahrhunderts als gesundes Gegenbeispiel zum urbanen, von Zucker und modernem Essen verdorbenen Gebiss der Stadtbevölkerung aufgefasst und erforscht wurde - und dass man diesen Gegensatz in koloniale Begriffe fasste: »Für ihre Dissertationen gingen etliche Zahnmediziner in die Alpen, um die Ernährungsweise der >Bergvölker $<$ zu studieren. Diese wurden gewissermassen als Pendant zu den >Naturvölkern < gesehen, die ebenfalls über auffallend gesündere Zähne verfügten als die Europäer. $\ll^{143}$

Die Seh- und Denkgewohnheiten, die mit solchen internen Schweizer Modernitätsdifferenzen verknüpft waren, wurden von Schweizer Forschenden auf ihre afrikanischen Forschungsobjekte übertragen, wie Patrick Harries schreibt: »In particular, they carried to Africa the European images, themes and attitudes employed to describe the Alps as a primitive wilderness. ${ }^{144}$ Ausgestattet mit diesen Bildern einer heimischen Wildheit machten sich Schweizer Forschende daran, deren »afrikanische Varianten « zu erkunden. Innerschweizerisches und außereuropäisches othering wurden dadurch unweigerlich miteinander verschränkt: Die Vorstellungen eines rückständigen Afrika, die beispielsweise durch die Vermittlung von Missionsgesellschaften großflächig in der Schweiz zirkulierten, stützten wiederum die Herausbildung einer Schweizer Identität, die sich als modern, entwickelt und hochtechnisiert verstanden wissen wollte. Harries führt dazu aus: »Missionary propaganda brought Africa directly into the intimate recesses of Swiss homes.

141 | Vgl. dazu auch den Beitrag von Martin Mühlheim für diesen Sammelband.

142 | Harries, "From the Alps to Africa", S. 219.

143 | Schär, "Karies", S. 104.

144 | Harries, "From the Alps to Africa", S. 201. 
In both private and public spaces, the picture of the dark continent served as a foil against which the Swiss could measure the evolution of their own society. «145 Weil nicht nur die Differenz zwischen der Schweiz und Afrika, Mittelland und Alpen, sondern auch Klassengegensätze oder die Geschlechterdifferenz mit dem Unterschied zwischen Natur und Kultur, Stillstand und Entwicklung, primitivem Leben und Zivilisation verknüpft wurden, können im Schweizer Selbstverständnis zahlreiche Parallelen, Überschneidungen und Verschränkungen zwischen diesen verschiedenen Prozessen des othering ausgemacht werden. ${ }^{146}$

\section{Koloniale Wissensproduktionen im SchWeizer Kontext}

Eine bedeutsame Aufgabe der postcolonial studies besteht darin, das Zusammenspiel von Wissenschaft und Herrschaft zu untersuchen. Dabei wird von einer gegenseitigen Konstitution von Wissen und Macht ausgegangen: Wissenschaftliche "Forschungen« wie die Rassentheorien des 19. Jahrhunderts stützen sich auf die Erkenntnisse, die Objekte und die Berichte der kolonialen Unternehmungen. Umgekehrt werden diese durch die wissenschaftliche Arbeit legitimiert. Und mehr noch: Der koloniale Blick, der sich dadurch auszeichnet, dass er andere Menschen zu Objekten macht, ${ }^{147}$ verdankt sich nicht zuletzt den diskursiven Bedingungen, welche die Wissenschaft mitetabliert: Sie macht grundlegende Differenzen mit Hilfe von Rassentheorien oder menschlicher Evolutionstheorien denkbar und scheinbar »einsichtig«. Für die postkoloniale Schweiz von Bedeutung ist, dass Schweizer Forschende und Institutionen in die Dynamik grenzüberschreitender Austauschprozesse und transkultureller Verhandlungen auf dem Felde der Anthropologie, Religionswissenschaft, Orientalistik und anderen wissenschaftlichen Gebieten eingebunden waren.

Eine Figur, die Expedition und Forschung verbunden hat, ist Carl Passavant, der aus großbürgerlichen und wohlhabenden Verhältnissen stammte. ${ }^{148}$ Auf seinen zwei Afrikareisen in den Jahren 1883 und 1884 machte sich Passavant auf die Suche nach dem »Unvermischten «, dem »Ursprünglichen«. Möglicherweise angeregt von Julius Kollmann - seit 1878 Professor in Basel und eine Kapazität auf dem Gebiet der Anatomie und physischen Anthropologie - entschied sich Passavant für das Thema seiner Dissertation Craniologische Untersuchungen der Neger und der Negervölker. Im ersten Satz seines Vorwortes heißt es darin: »Bei einer Umschau über die auffallenden Abarten des Menschengeschlechtes, welche der afrikanische Continent beherbergt, scheint es mir geboten, dass der Craniologe seine Stellung zu

$145 \mid$ Harries, Butterflies \& Barbarians, S. 4.

146 | Vgl. dazu den Beitrag von Bernhard Schär in diesem Band.

147 | Vgl. Fanon, Weisse Masken.

148 | Die nachfolgenden Ausführungen stützen sich auf Keller, "Sieben Schädel«; Schneider/Lüthi, "Passavant“; Schneider/Röschenthaler/Gardi, Fotofieber. 
der Rassenfrage darlege. ${ }^{149}$ Die physische Anthropologie rückte den Körper - Statur, Kopfform, Pigmentierung - und die mentalen Kapazitäten anderer Völker als Untersuchungsobjekte in den Mittelpunkt ihres Interesses. Sie erhoffte damit, zur Klärung der Abstammungsfrage und der »Rassen« beizutragen. Passavant stützte seine Thesen auf eine Zusammenstellung von 205 Schädeln unterschiedlicher Provenienz; nur gerade sieben davon hatte er selber vermessen. Den Rest seines Datenmaterials entnahm er den zahlreichen Untersuchungen anderer Anthropologen. Unklar bleibt, wie er zu seinen Kriterien für die Klassifikation der Schädel in die Kategorien »Nigriter«, »Congovölker«, »Kaffer«, »Hottentotten« und »Buschmänner « gelangte. In einer Fotografie, die wahrscheinlich von seinem Bruder angefertigt wurde und welche die Überschrift Neger-Schädel zur Doctor-Dissertation von Karl Passavant trägt, sind auch einige Affenschädel zu sehen. ${ }^{150}$

Die Schädelvermessungen nahmen spätestens seit der Aufklärung vor allem innerhalb der physischen Anthropologie und mit Hilfe der Anthropometrie bei der Bestimmung der Wertigkeit von Menschengruppen einen wichtigen Platz ein. ${ }^{151}$ Sie standen in einer langen Tradition (erinnert sei hier an den Zürcher Johann Kaspar Lavater), in der mit zunehmendem Einfluss die Medizin, Anthropologie und Biologie im 18. Jahrhundert der Entstehung der menschlichen Gattung und den Gründen für die unterschiedliche Entwicklung einzelner Menschengruppen nachspürten. Bereits früh tauchten in diesem Zusammenhang Fragen auf, die spätere Rassendoktrinen prägen würden: Gibt es einen Zusammenhang zwischen körperlichen Merkmalen und psychisch-geistiger Veranlagung? Trotz des Versuchs, eine scheinbar objektive Theorie menschlicher Differenz zu etablieren, beinhaltete die Praxis in der Medizin und der physischen Anthropologie eine höchst subjektive und rassistische Vorgehensweise in der Beurteilung der »Menschenrassen«. Geprägt waren diese Wissenschaften von einer biologisch deterministischen Sprache und einem methodologischen Reduktionismus. Ihr Ausgangspunkt war die unhinterfragbare Überlegenheit der »europäischen Rasse« gegenüber den Nichteuropäerinnen, -europäern und marginalisierten Gruppen innerhalb der eigenen Gesellschaften. ${ }^{152}$

Auch bei Passavant dienten die wissenschaftliche Arbeit und das Sammeln der Fotos als Beweis für die Überlegenheit der »eigenen« Kultur. Sie sind ein wichtiges Zeugnis davon, wie Afrika aus europäischer Sicht gesehen werden sollte; zugleich dienten sie der Repräsentation der eigenen zivilisatorischen Vormachtstellung. Ähnlich wie Passavant machte sich der Zürcher Professor Otto Schlaginhaufen einige Jahrzehnte später (und nachdem er auf Melanesien zahlreiche Menschen vermessen und 420 Schädel gesammelt hatte) auf die Suche nach dem rassischen

149 | Passavant, "Craniologische Untersuchung", zit. n. Keller, "Sieben Schädel«, S. 43.

150 | Ebd., S. 48.

151 | Zum Beitrag der Anthropologie zum Rassedenken siehe Priester, Rassismus, S. 60ff. 152 | Vgl. dazu auch die Aufarbeitung des Rassismus in den Schriften des Schweizer Psychiaters August Forel in Bugmann/Sarasin, "Forel mit Foucault". 
Ursprung der Schweizer, der mit dem »Homo alpinus helveticus « bereits im 18. Jahrhundert von Johann Jacob Scheuchzer begrifflich gefasst worden war. ${ }^{153}$

Ein anderer wichtiger Kontext, in dem koloniales Wissen entstand und zirkulierte, stellen die bereits erwähnten Missionsgesellschaften dar. Die Untersuchung von Patrick Harries verdeutlicht, dass nicht nur die »Zivilisierungsmission« die europäischen Kolonialmächte antrieb, sondern dass die kolonisierten Orte einen Schauplatz für die Suche nach einem verlorenen, ursprünglichen (und oftmals »urchristlichen«) Leben jenseits von Materialismus und Immoralität darstellten. ${ }^{154}$ Auch Schweizer Missionaren und ihren Gemeindemitgliedern erlaubte Afrika derart, in eine vermeintlich intakte, unverdorbene Welt einzutreten, frei von den Konflikten und Entzauberungen des industriellen Zeitalters. Auf ein solches, mystifizierendes Bild stützt sich zum Teil auch die missionarische Kritik am Imperialismus, wonach dieser Kriege, Alkohol und eine lasterhafte Zivilisation aus Europa nach Afrika bringen würde.

Die Rückwirkungen der missionarischen Arbeit in Afrika auf die Schweiz waren vielfältig und dokumentieren, wie sich ein koloniales »Alltagswissen « etablieren und verbreiten konnte. Die Sammelarbeit und Missionspropaganda brachte Bilder von Afrika auf direktem Wege in die intimsten Winkel von Schweizer Heimstätten: Vorträge von heimkehrenden Missionaren, Predigten, Sonntagsschulen, Museumssammlungen, botanische Gärten wie auch eine Ansammlung an Fotografien und Gedenkalben trugen zur Verbreitung dieses Wissens über Afrika bei.

Durch die transnationale Organisation bedeutsamer Missionsgesellschaften wie etwa der Basler Mission, zirkulierte über das Medium von Missionszeitschriften auch unverhohlene koloniale Propaganda in Schweizer Haushalten. So erscheint im Evangelischen Missions-Magazin der Basler Mission von 1898 ein Artikel mit dem Titel »Die Bedeutung der Mission für unsere Kolonien«, in dem es heißt:

"Wir alle fühlen die Verpflichtung, die uns durch unsere Kolonien mit ihren Millionen heidnischer Bevölkerung auferlegt ist. Es ist zwar nicht jedermanns Sache, an den anderen Bemühungen zur Erschließung derselben, an den kommerziellen, militärischen und wissenschaftlichen Arbeiten beizutragen. Aber zu dieser segensvollen und zentralsten aller Arbeiten zum Segen unserer [deutschen] Schutzgebiete, zur Mission können und sollen Sie alle mithelfen. «155

Dass die Aufarbeitung der kolonialen Episteme für die Schweiz nicht nur von historischem Interesse ist, soll ein abschließendes Beispiel verdeutlichen: In Schweizer Museen lagern zahlreiche Kult- und Kunstgegenstände sowie menschliche Schädel und Knochen, die in einem kolonialen Kontext und oft unter unlauteren Bedingungen erworben worden sind. Es ist vermutlich eine Frage der Zeit, bis die

153 | Vgl. dazu Keller, Der Schädelvermesser, S. 51ff. und S. 86.

154 | Patrick Harries, Butterflies \& Barbarians.

155 | Richter, "Die Bedeutung der Mission", S. 323. 
Restitution dieser Objekte mit den ethischen, religiösen, ästhetischen und ökonomischen Aspekten, die diese mit sich bringt, auch hierzulande zum Thema wird. ${ }^{15} 6$

\section{Postkolonialer Widerstand}

So sehr die postkoloniale Theorie darum bemüht ist, die Zusammenhänge zwischen Wissen, gesellschaftlicher Organisation und Macht aufzuzeigen, so wichtig ist es für sie auch, Widerstand denkbar zu machen und zu lokalisieren. Homi Bhabha hat dafür den Begriff der Hybridität ins Feld geführt. Koloniale Machtansprüche werden angreifbar, so seine These, wenn erkannt wird, dass die Asymmetrie zwischen dem herrschenden Subjekt und dem kolonisierten Anderen ständig hergestellt und aufrechterhalten werden muss. Die Reproduktion kolonialer Herrschaft ist somit begleitet von zahlreichen Störungen, Brüchen, Ungereimtheiten und einem phantasmagorischen Überschuss, die den Bereich des Hybriden eröffnen. Dieser ermöglicht einerseits Einblicke in die Konstruiertheit und Schwachstellen kolonialer Macht. Andererseits erweist er sich als Einsatzstelle für subversive Praktiken, die sich gegen die vorherrschende Macht wenden und sie anfechten. Diese Veränderung der Perspektive hat zur Folge, dass »die Wirkung der kolonialen Macht in der Produktion von Hybridisierung [...] statt in der lautstarken Ausübung der kolonialistischen Autorität oder der stillschweigenden Unterdrückung einheimischer Tradition ${ }^{157}$ gesehen wird. Diese Einsicht, die Bhabha anhand der Analyse von Beispielen aus dem kolonialen Indien gewinnt, hat sich für die postkoloniale Theorie als folgenreich erwiesen. So sehen Hannah Franzki und Joshua Kwesi Aikins auch gegenwärtig eine Hauptaufgabe der postkolonialen Studien darin, »zu einer Analyse aktueller Machtverhältnisse unter Berücksichtigung ihrer kolonialen Ursprünge bei[zu]tragen, die gleichzeitig Ausgangspunkt für politischen Widerstand sein kann $\ll^{158}$.

Widerstand kann sich auch, wie im Folgenden gezeigt wird, in der Umdeutung einer rassistischen Kampagne durch eine alternative Bildpolitik manifestieren. Ausgangspunkt ist eine Kampagne der Schweizerischen Volkspartei (SVP), die 2007 für die Annahme der von ihr lancierten sogenannten Ausschaffungsinitiative warb. Die Initiative verlangte die Ausweisung von Ausländerinnen und Ausländern, die in bestimmter Weise straffällig geworden sind, und wurde am 28. November 2010 mit 52,9 Prozent der Stimmen angenommen. Das Plakat, das für die Initiative warb und weit über die Landesgrenzen hinaus Anlass zu Diskussionen

156 | Siehe dazu die Konferenz Mémoire africaine en péril. Pillages et restitutions du patrimoine culturel et anthropologique africain, die im Juni 2011 am Musée d'art et d'histoire in Genf stattfand. In Bezug auf Frankreich siehe die Stellungnahme von Nicolas Bancel unter http://www.infoclio.ch/fr/node/23795, 06.06.2011.

157 | Bhabha, Verortung, S. 166.

158 | Franzki/Aikins, "Postkoloniale Studien“, S. 16. 
gab, zeigt ein schwarzes Schaf, das von einem weißen aus der »Schweiz« - symbolisiert durch eine rote Fahne mit Schweizerkreuz - gekickt wird. Die problematische Bildsprache des »Schäfchenplakats« blieb kein Einzelfall. Es kann vielmehr als Auftakt einer Kampagnenserie betrachtet werden, die gezielt rassistische Inhalte zum Einsatz bringt.

Abbildung 2: Abstimmungsplakat der SVP zur Ausschaffungsinitiative 2007

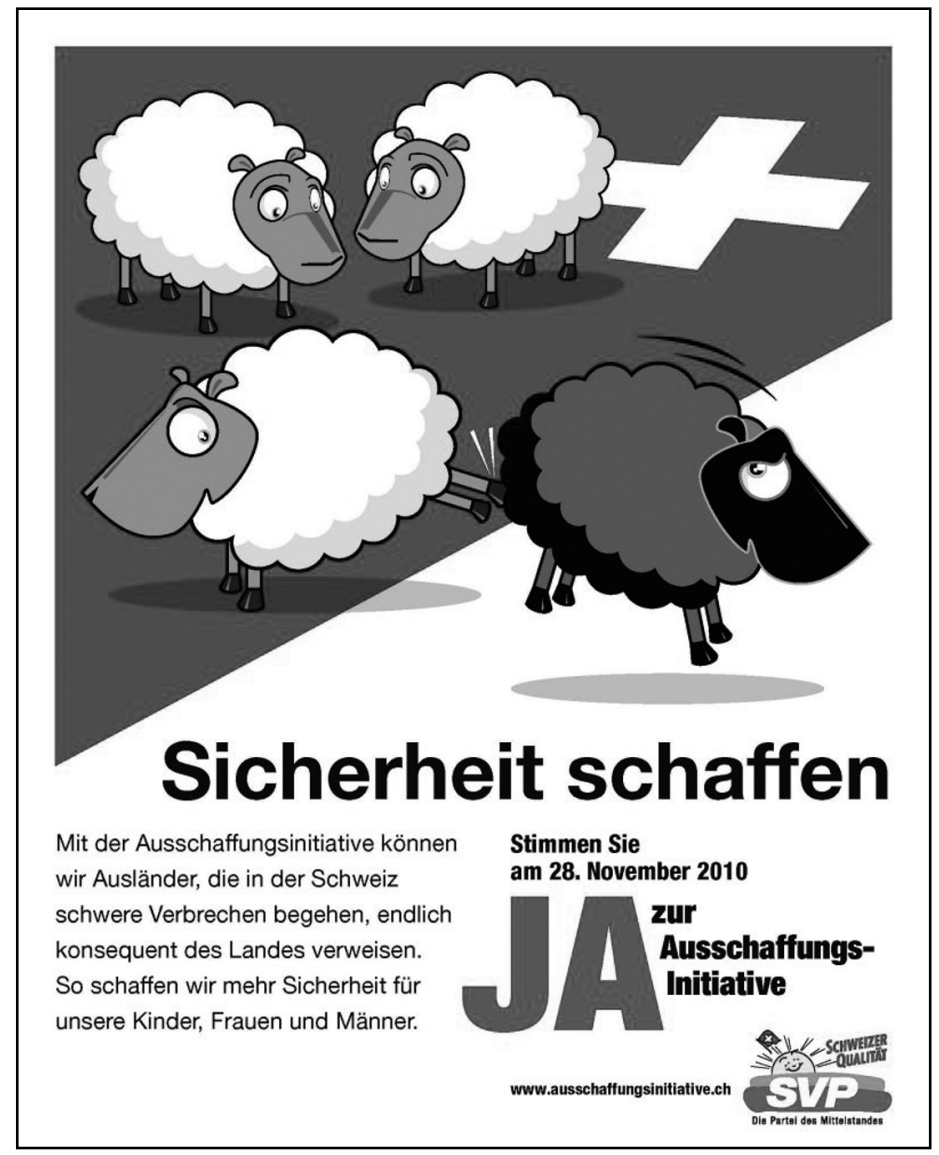

Indem das Plakat so verstanden werden kann, dass Dunkelhäutige in der Schweiz die schwarzen Schafe seien, spielt das Plakat mit der Mehrdeutigkeit der Metapher des schwarzen Schafs: Einerseits operiert es mit rassistischen Elementen, die klar erkennbar sind, andererseits konnte sich die SVP auf die Position zurückziehen, dass hier nur die Redensart gemeint sei. Solche Bilder machen insofern Politik, als dass sie auf diese Weise die Grenzen des Sag- und hier vor allem Zeigbaren 
verschieben. ${ }^{159} \mathrm{Im}$ Kontext der postkolonialen Schweiz stellt sich die Frage, ob die größte Schweizer Regierungspartei auf eine solche Bildsprache zurückgreifen kann, weil die Schweiz als Ort gilt, der von historischen Rassismen unbelastet sei und sich außerhalb kolonialer Konstellationen befinde. ${ }^{160}$ Der Bericht des UNOSonderberichterstatters für Rassismus hielt in diesem Zusammenhang fest, bedenklich sei in Bezug auf die Schweiz die Tatsache, dass rassistische Ideen auch auf demokratische Parteien übergriffen. Der Transfer von diesem Gedankengut in politische Abstimmungen und Wahlkämpfe sei Besorgnis erregend. ${ }^{61}$ Solche Rassismen würden jedoch selten thematisiert, hält Noémi Michel in Bezug auf die sogenannte Anti-Minarett-Initiative fest, welche den Bau von Minaretten in der Schweiz verbietet und am 29. November 2009 von 57,5 Prozent der Schweizer Stimmbevölkerung angenommen worden ist. ${ }^{162}$

Das Schaf der »Ausschaffungsinitiative« wurde von der Gegenseite in subversiver Absicht aufgenommen und modifiziert, unter anderem vom Verein Moutons de Garde, der als Reaktion auf die Kampagne 2007 ins Leben gerufen wurde und bis Ende 2010 aktiv war. Er wollte keine bestimmte politische Position vertreten, sondern sich für eine parteiübergreifende Diskussionspolitik aussprechen, die auf Respekt basiert. Der Onlineaufruf der Initiative, der sich gegen »jedes Vorgehen [richtet], das Hass, Angst, Rassismus und Fremdenfeindlichkeit schürt « ${ }^{16_{3}}$, wurde von über 33.000 Personen unterzeichnet. Das zweite Beispiel fordert zur Identifikation mit dem schwarzen Schaf auf, das sich nicht leichthin aus der Schweiz kicken lässt. Es diente als Emblem eines Festes gegen Rassismus, das am 6. Oktober 2007 in Bern, zeitgleich mit einer Demonstration der SVP, stattfand. Das Aufeinanderprallen von Demonstration und Gegendemonstration führte zu einer gewalttätigen Konfrontation; über die damaligen Ereignisse wurde weltweit berichtet. ${ }^{164}$ Diese subversiven Verwendungen der Schafe machen unterschiedliche Strategien des Widerstands kenntlich; die beiden Vorgangsweisen symbolisieren nicht zuletzt eine unterschiedliche Einschätzung im Umgang mit der SVP. Beim zweiten Fall mutiert das »Opferlamm« zum wutschnaubenden Tier: Das schwarze Schaf erscheint als widerständiges und zorniges Wesen, das die Konfrontation mit rassisti-

159 | Bischoff/Falk/Kafehsy, "Images".

160 | Zum Zusammenhang von Rechtspopulismus und Migrationspolitik siehe Skenderovic/D’Amato, Mit dem Fremden politisieren.

161 | Diène, "Mission to Switzerland". Siehe dazu den Beitrag von Franziska Jenni und Francesca Falk in diesem Band.

162 | "Il est intéressant de noter que les partis et associations qui ont mené campagne contre l'initiative anti-minaret n'ont que rarement condamné cette initiative pour son caractère raciste «. Onlineinterview von Elsa Dorlin mit Noémi Michel vom 30.11.2009 publiziert auf dem Blog http://observatoire2.blogs.liberation.fr/normes_sociales/2009/11/ des-minarets-aux-miradors-entretien-avec-no \%C3 \%A9mi-michel.html, 17.07.2011.

163 | Vgl. http://www.moutonsdegarde.ch, 23.02.2011.

164 | Vallely, "Switzerland «; Sciolino, "Immigration ". 
Abbildungen 3 und 4: Motiv des Vereins Moutons de Garde und Emblem des Festes gegen Rassismus
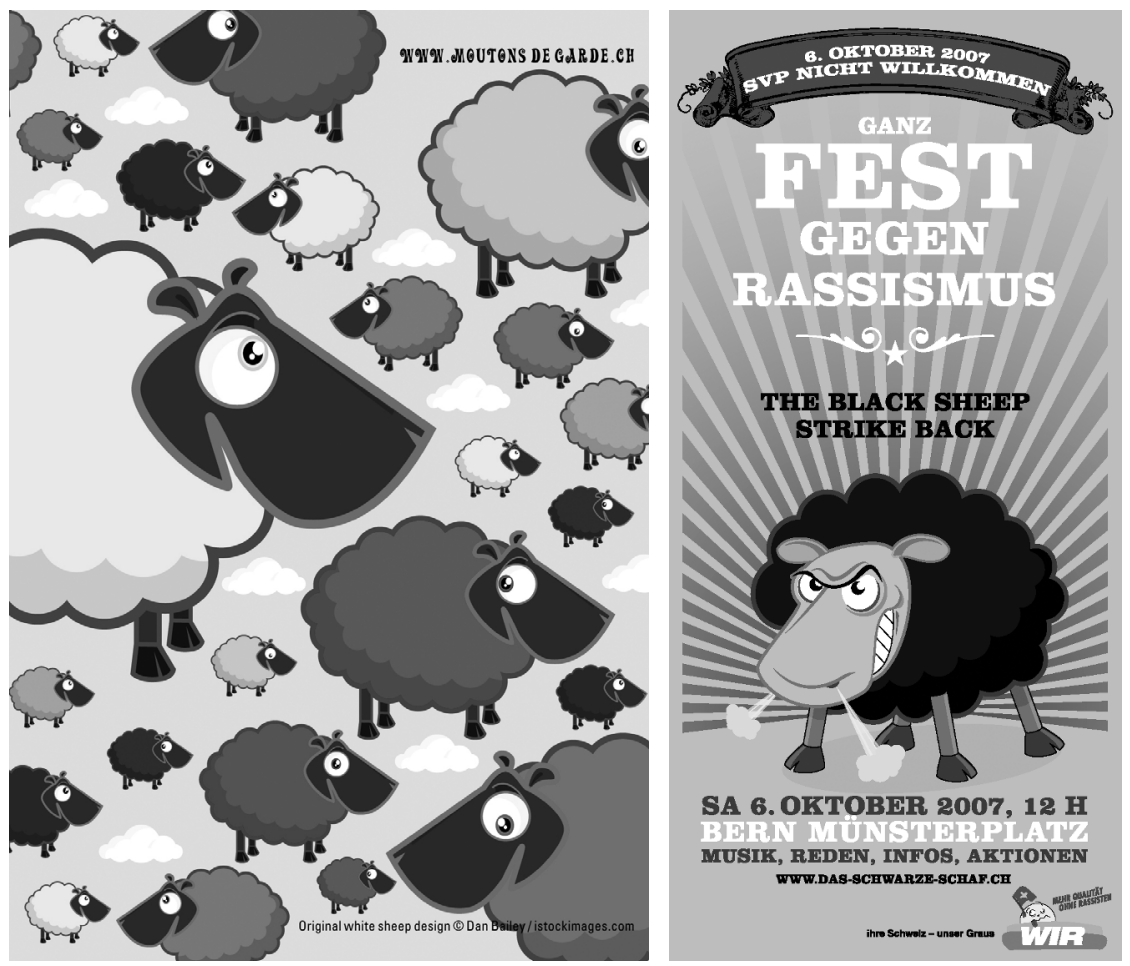

schen Kräften nicht scheut. Es vertritt eine Position, die es aufgrund ihres Verständnisses verneint, mit rassistisch agierenden Parteien wie der SVP das Gespräch zu suchen oder gar Kooperationen einzugehen. Hier wird der Aufruf nach Ausgrenzung mit eigenen Grenzziehungen beantwortet - »SVP nicht willkommen« liest sich der Text zum Bild. Beim ersten Beispiel folgen die bunten Schafe einer Logik der Multikulturalität: »Wir sind alle farbige Schafe«, wird als Botschaft transportiert. Zugleich soll fremdenfeindliche Politik nicht widerspruchslos weitergeführt werden können: Nicht der Wachhund, sondern die Wachschafe schützen dabei die Schweiz vor Fehlentwicklungen. Sowohl die weißen als auch die schwarzen Schafe, die gleichsam die Polarisierung der politischen Diskussion symbolisieren, verschwinden in der Darstellung der Moutons de Garde. Auf die aggressive Abstimmungskampagne wird in diesem Fall mit der Aufforderung nach Deeskalation und einem friedlichem Zusammenleben in einer pluralen Gesellschaft reagiert - gekoppelt allerdings mit der Überwachung fremdenfeindlicher Positionen.

Die Aufforderung, hegemoniale, rassistische und eurozentrische Darstellungen anders denn nur als Ausdruck von Macht und Überlegenheit zu lesen, heißt demnach, aufmerksam zu bleiben für Zäsuren, Gegenläufigkeiten, Unstimmig- 
keiten, Selbstwidersprüche und auch für die Leerstellen und das Ungenannte. Es bedeutet aber auch, das postkoloniale Archiv mit anderen Stimmen, Gegendiskursen, mit den kritischen und subversiven Positionen anzureichern, die es auch gibt und gegeben hat. Das können literarische Stimmen wie diejenige von Mariella Mehr oder Martin R. Dean sein. ${ }^{165}$ Es kann die Arbeit von Gruppierungen sein, die den gängigen Stereotypen andere Formen der Selbstrepräsentation entgegensetzen - wie dies etwa im Kontext des Black Women's Center Zürich oder Sankofa. Plattform für Menschen afrikanischen Erbes geschieht. ${ }^{166}$ Es kann die Aktivität der bereits erwähnten NGOs sein, welche die Verschränkung der Schweizer Politik und Wirtschaft mit postkolonialen Strukturen aufzeigen. Oder diejenige der Menschenrechtsorganisation augenauf, die sich für die Rechte von Migrantinnen und Migranten einsetzt und dabei insbesondere die Arbeit von Polizei und Behörden kritisch begleitet. ${ }^{167}$ Als Beispiel für postkolonialen Widerstand kann schließlich auch die von Hans Fässler und Sasha Huber initiierte Petition zur Umbennenung des Agassizhorns bezeichnet werden, die von 2500 Personen unterzeichnet worden ist. Sie weist darauf hin, dass der in der Schweiz vor allem für seine glaziologische Forschung bekannte Louis Agassiz »einer der wichtigsten Wegbereiter des so genannten >wissenschaftlichen $<$ Rassismus und ein Vordenker der Apartheid ${ }^{168}$ gewesen sei. Die Petition schlägt vor, den nach ihm benannten Gipfel nach einem Sklaven, von dem Agassiz im Rahmen seiner Rassenforschung Fotografien anfertigen ließ, fortan als Rentyhorn zu bezeichnen. ${ }^{169}$

165 | Siehe dazu der Beitrag von Alexander Honold in diesem Band. Vgl. auch Bröck, "Slavery, Race and Postcolonial Love".

166 | Siehe die Webseiten der Organisationen: http://www.blackwomenscenter.ch; http://www.sankofa.ch, 22.04.2011.

167 | Vgl. http://www.augenauf.ch, siehe auch die Webseite der Fachstelle Frauenhandel und Frauenmigration: http://www.fiz-info.ch, 22.04.2011.

168 | Vgl. http://www.rentyhorn.ch sowie http://www.louverture.ch und http://www.sasha huber.com, 22.04.2011.

169 | Die drei Gemeinden Grindelwald, Guttannen und Fieschertal, auf deren Boden sich das Agassizhorn befindet, haben in einem Schreiben vom Juli 2010 verlauten lassen, dass sie den Vorstoß ablehnen und auch keinen namenlosen Nachbargipfel des Agassizhorns Rentyhorn taufen wollen. Die Ablehnung begründet der Gemeindepräsident von Grindelwald mit einer Bemerkung, die für den helvetischen Umgang mit dem Thema als charakteristisch gelten kann. Agassizs Rassentheorien würde man aus heutiger Sicht zwar klar verurteilen, sagt er, jeder Mensch habe jedoch "Sonnen- und Schattenseiten". Wälti, "Agassizhorn". 


\section{Postkoloniale Schweiz: \\ WEITLÄUFIGE FORSCHUNGSFELDER \\ UND ALLTAGSPOLITISCHE DRINGLICHKEITEN}

Diese Einleitung stellt auch den Versuch einer Bestandesaufnahme dar. Die Anzahl zitierter Forschungsberichte macht deutlich, dass für zahlreiche Themen, die für die postkoloniale Schweiz von Bedeutung sind, bereits Forschungsresultate vorliegen. Mit Methoden und Ansätzen der postkolonialen Theorie wurde dabei allerdings kaum oder nur am Rande gearbeitet. Wie die Aufsätze im vorliegenden Band zeigen und wie unsere Ausführungen in dieser Einleitung deutlich machen sollen, lassen sich mit Hilfe der postkolonialen Perspektive neue Zugänge zur Schweiz und ihrer Kontextualisierung in einer globalisierten Welt und in der Geschichte der Moderne erschließen.

Neben dem Umschreiben der bestehenden Forschung, zu der wir anregen möchten, ist es auch nötig, den Forschungslücken Beachtung zu schenken: Auffallend ist etwa, dass es kaum Forschung zum Schweizer Kontext gibt, welche die Verschränkung von Kolonialismus mit Geschlecht und Sexualität in den Blick nimmt. Feministische und queere Perspektiven auf die postkoloniale Schweiz könnten an vielen Punkten ansetzen: Inwiefern haben sich Schweizer Frauen, beispielsweise im Rahmen von Missions- oder Handelstätigkeiten, an der Verbreitung und Durchsetzung kolonialer Praktiken und Weltsichten beteiligt? ${ }^{370}$ Wie sind Konzepte von Schweizer Männlichkeiten wie etwa dem technisch versierten Ingenieur mit postkolonialen Formen des othering verschränkt? Inwiefern gründen Repräsentationen weißer Weiblichkeiten und Männlichkeiten in der Schweiz auf der impliziten und expliziten Abgrenzung von nicht weißen Anderen? ${ }^{171}$ Wie können etwa die Entstehungsbedingungen und Resonanzen von Corinne Hofmanns ungemein erfolgreicher autobiographischen Schilderung »Die Weiße Massai« in Bezug zur postkolonialen Schweiz gesetzt werden ${ }^{172}$ Vermehrt diskutiert wird in jüngerer Zeit auch die Instrumentalisierung von Frauenrechten für rassistische und insbesondere islamfeindliche Politiken. Aus feministisch-postkolonialen Kreisen in Deutschland wird etwa die wachsende Beliebtheit von Alice Schwarzer kritisiert. Lange als »Radikalfeministin « verschrien, erweisen sich ihre islamkritischen Positionen als anschlussfähig an eine Diskussion, welche die (westlichen) Frauenrechte gegen ein (östliches) Patriarchat auszuspielen suchen. ${ }^{173}$ »Mainstream-Feminismus wird hier zum direkten Agenten einer fremdenfeindlichen neo-orientalistischen

170 | Vgl. dazu die in Deutschland erschienenen Studien Walgenbach, Die weiße Frau, sowie Dietrich, Weiße Weiblichkeiten.

171 | Vgl. Iso, "Weiss - wie Schneewittchen". Ein wegweisender Versuch, die whiteness studies mit der Schweiz zu verknüpfen, wurde kürzlich von Noémi Michel und Manuela Honegger unternommen. Vgl. Michel/Honegger, "Thinking Whiteness".

172 | Vgl. dazu Krüger, "Die Weisse und die edlen Wilden ".

173 | Dazu der hier publizierte Artikel von Meral Kaya. 
Politik«, hält Gabriele Dietze diesbezüglich fest. ${ }^{174}$ Solche »Entlastungsdiskurse« verdecken zudem eigene Defizite in Bezug auf die Gleichstellung der Geschlechter. Vergleichbare Tendenzen lassen sich, wie eine Themennummer der Zeitschrift Olympe unlängst dokumentiert hat, auch in der Schweiz feststellen. ${ }^{175}$

Ähnliches ließe sich zudem für die Instrumentalisierung von lesbian and gay rights festhalten: Auch der Schutz sexueller Minderheiten wird dazu verwendet, die Toleranz westlicher Gesellschaften in Absetzung von anderen zu behaupten. ${ }^{176}$ Eine solche Sicht blendet die nach wie vor bestehenden Diskriminierungen queerer Menschen in westlichen Gesellschaften aus ${ }^{177}$ und stellt diese in einen scharfen Kontrast zu angeblich homophoben und archaischen nicht westlichen Traditionen. Diese Differenz trennt nicht nur westliche von nicht westlichen Kulturen, sie organisiert die westlichen Migrationsgesellschaften auch in ihrem Inneren: Sie wird benutzt, um den Unterschied zwischen einer vormodernen, »verschlossenen« und einer modernen, »aufgeschlossenen« Bevölkerung herzustellen. Dieses plakative Vorführen einer liberalen Haltung des Westens gegenüber queeren Menschen, welche auf der Abgrenzung und Stigmatisierung von Migrantinnen und Migranten, insbesondere muslimischer Herkunft, basiert, bezeichnet Jasbir Puar als »Homonationalismus $«^{178}$. Inwiefern lässt sich dieses Konzept auch mit der postkolonialen Schweiz in Verbindung bringen? ${ }^{179}$ Aus einer queer-feministischen Optik ist auch die Frage von Bedeutung, ob und wie die zunehmende Inklusion von Lesben und Schwulen (etwa durch das 2007 in Kraft getretene Partnerschaftsgesetz) mit der gleichzeitigen Verschärfung der Asyl- und Ausländerpolitik (gerade auch im Heiratsrecht) gekoppelt ist. Fatima El-Tayeb hat bereits 2005 für Deutschland festgehalten, dass dieses »Anbieten der Option bürgerlicher >Normalität< für sexuelle Außenseiter einher[geht] mit einem Erstarken eines Modells kulturalistischer Anomalität «, ${ }^{180}$ das queers of color ganz besonders (be-)trifft. Lässt sich diese Analyse auch auf die Schweiz übertragen - und welche Folgen zeitigt eine solche Analyse für queer-feministische Forschung und Politik?

Untersucht werden müsste ferner, wie sich Rassismus in Schweizer Kontexten mit Antisemitismus sowie unterschiedlichen Formen der Fremdenfeindlichkeit, beispielsweise gegenüber sogenannten »Gastarbeiterinnen und Gastarbeitern«,

174 | Dietze, "Critical Whiteness", S. 237.

175 | Vgl. Olympe, Wider die Instrumentalisierung.

176 | Lüthi, "Coming to Terms".

177 | Dass solche Diskriminierungen keineswegs der Vergangenheit angehören, belegt etwa das gesetzliche Verbot der Adoption und Stiefkindadoption für gleichgeschlechtliche Paare in der Schweiz, welche das Leben queerer Familien erheblich beeinträchtigt.

178 | Puar, Terrorist Assemblages.

179 | Zu schweizerischen Versionen des Homonationalismus vgl. die Kommentare von Eveline Y. Nay und Sushila Mesquita in Purtschert, "Chewing on Post_colonial Switzerland, Part II", sowie Mesquita, Ban Marriage!

180 | El-Tayeb, "Begrenzte Horizonte«, S. 133. 
verschränkt, überlagert und verbindet. Ein weiteres Forschungsdesiderat besteht in der Untersuchung einer postkolonialen Schweiz, die den Sprachregionen gebührend Beachtung schenkt. Der vorliegende Band erscheint auf Deutsch und beschäftigt sich verstärkt mit Beispielen aus der deutschsprachigen Schweiz. Eine systematisch vergleichende Perspektive, die postkoloniale Konstallationen in unterschiedlichen Gebieten vergleichen könnte, kann damit nicht eingenommen werden. Es ist anzunehmen, dass die transnationalen Verbindungen zwischen franko-, germano- und italophonen Ländern sowie den entsprechenden Teilen der Schweiz wichtige Unterschiede in den jeweiligen Regionen zur Folge haben. So sind Konzepte und Vorstellungen, die für die französischen und belgischen Kolonien von Bedeutung waren, durch den verstärkten Austausch von Informationen, wissenschaftlichen Ergebnissen und medialen Erzeugnissen im frankophonen Sprachraum vermutlich in der französischsprachigen Schweiz stärker präsent gewesen, während sich die kolonialen Bilder und Begriffe der deutschsprachigen Schweiz stärker im Austausch mit Deutschland herausgebildet haben; die gleiche Vermutung ließe sich auch für das Tessin und die italienischen Kolonien in den Raum stellen. Auch die Frage, was die Anwendung einer solchen, sprachgebundenen Perspektive auf den Postkolonialismus der rätoromanischen Schweiz zutage bringen würde, kann an dieser Stelle nur aufgeworfen werden.

In der Schweiz ist, so lässt sich abschließend festhalten, eine eklatante »Leerstelle« zu erkennen, wenn es um Fragen des Rassismus, seiner kolonialen Genealogie und seiner gesellschaftlichen Auswirkungen geht. Einer solchen allgemeinen Nichtbeachtung stehen Einschätzungen wie die von Carmel Fröhlicher-Stines und Kelechi Monika Mennel gegenüber, die in der Einleitung ihres Berichts zur Situation von schwarzen Menschen in der Schweiz schreiben: »Stereotype Bilder, die auf die Schwarzen projiziert werden, stammen zum grossen Teil aus der Zeit der Beziehungen zwischen Europa und Afrika, die von Kolonisation und Sklaverei geprägt war. Von diesen Vorstellungen sind noch heute erst vor kurzem zugewanderte sowie längst einheimische Schwarze betroffen. ${ }^{181}$ Eine postkoloniale Perspektive vermag es, solche zur Selbstverständlichkeit geronnenen Vorstellungen aufzubrechen, durch die der strukturelle Rassismus in der Schweiz gleichzeitig omnipräsent und unsichtbar gemacht wird. Indem die Eingebundenheit der Schweiz in transnationale koloniale Systeme zum Thema gemacht wird, kann aufgezeigt werden, wie rassistische Vorstellungen zirkulieren und wie sie naturalisiert oder kulturalisiert werden. Wenn also eine Auseinandersetzung mit Fragen des »Postkolonialen« für die Schweiz gefordert wird, steckt mehr dahinter als ein moralisierender Aufruf oder eine akademische Modeströmung. Auch wenn es situativ Sinn ergibt, eine klare Unterscheidung zwischen territorialen Kolonialmächten und anderweitig in den Kolonialismus involvierten Staaten zu treffen, ist eine

181 | Fröhlicher-Stines/Mennel, Schwarze Menschen, S. 9. Vgl. auch Matare/Schneider/ Zeugin, Black, Noir, Schwarz. 
eingehende Beschäftigung mit der postkolonialen Schweiz und ein transnationaler Austausch über »Kolonialismus ohne Kolonien« überfällig geworden.

\section{LITERATURVERZEICHNIS}

Ahmad, Aijaz, In Theory. Classes, Nations, Literatures, London 1992.

Albertini, Rudolf/Wirz, Albert von, »Kolonialismus«, in: Historisches Lexikon der Schweiz, http://www.hls-dhs-dss.ch/textes/d/D26457.php, 28.10.2008.

Aldrich, Robert, »Le musée colonial impossible«, in: Blanchard, Pascal/Bancel, Nicolas (Hg.), Culture post-coloniale 1961-2006, Paris 2005, S. 83-91.

Amodeo, Immacolata, »In the Empire's Eyes: Africa in Italian Colonial Cinema between Imperial Fantasies and Blind Spots«, in: Fischer-Tiné, Harald/Gehrmann, Susanne (Hg.), Empires and Boundaries. Race, Class, and Gender in Colonial Settings, New York 2009, S. 166-178.

Andall, Jacqueline/Duncan, Derek, »Memories and Legacies of Italian Colonialism«, in: dies. (Hg.), Italian Colonialism, S. 9-28.

Anderson, Benedict, Imagined Communities. Reflections on the Origin and Spread of Nationalism, London 1991.

Anette Dietrich, Weiße Weiblichkeiten. Konstruktionen von »Rasse« und Geschlecht im deutschen Kolonialismus, Bielefeld 2007.

Arendt, Hannah, Elemente und Ursprünge totaler Herrschaft. Antisemitismus, Imperialismus, Totalitarismus, München 2008.

Bachmann-Medick, Doris, Cultural Turns. Neuorientierungen in der Kulturwissenschaft, Hamburg 2009.

Badenberg, Nana, »Die Bildkarriere eines kulturellen Stereotyps«, in: Honold, Alexander/Scherpe, Klaus R. (Hg.), Mit Deutschland um die Welt. Eine Kulturgeschichte des Fremden in der Kolonialzeit, Stuttgart 2004, S. 173-182.

Balibar, Etienne/Wallerstein, Immanuel, Rasse, Klasse, Nation. Ambivalente Identitäten, Hamburg 1992.

Bancel, Nicolas, »Introduction«, in: ders. et al. (Hg.), Ruptures postcoloniales. Les nouveaux visages de la société française, Paris 2010, S. 9-36.

Bancel, Nicolas/Bernault, Florence/Blanchard, Pascal/Boubeker, Ahmed/Mbembe, Achille/Vergès, Françoise (Hg.), Ruptures postcoloniales. Les nouveaux visages de la société française, Paris 2010.

Bancel, Nicolas/Blanchard, Pascal, »Avant-Propos. Culture post-coloniale: Le temps des héritages«, in: dies. (Hg.), Culture post-coloniale 1961-2006, Paris 2005 , S. 6-21.

Behrendt, Richard Fritz, Die Schweiz und der Imperialismus. Die Volkswirtschaft des hochkapitalistischen Kleinstaates im Zeitalter des politischen und ökonomischen Nationalismus, Zürich 1932.

Betulius, Walter, Friedrich Salomon Vögelin 1837-1888. Sein Beitrag zum schweizerischen Geistesleben in der zweiten Hälfte des 19. Jahrhunderts, Winterthur 1956. 
Bhabha, Homi, Die Verortung der Kultur, Tübingen 2000.

Bhambra, Gurminder K., Rethinking Modernity. Postcolonialism and the Sociological Imagination, Basingstoke 2009.

Bischoff, Christine/Falk, Francesca/Kafehsy, Sylvia (Hg.), Images of Illegalized Immigration. Towards a Critical Iconology of Politics, Bielefeld 2010.

Blanchard, Pascal/Bancel, Nicolas/Lemaire, Sandrine (Hg.), Culture coloniale en France, Paris 2008.

Dies. (Hg.), La fracture coloniale. La société française au prisme de l'héritage colonial, Paris 2005 .

Brändle, Rea, Wildfremd, hautnah. Völkerschauen und Schauplätze. Zürich 18801960, Zürich 1995.

Bröck, Sabine, »Slavery, Race and Postcolonial Love: The Swiss Novel >Die Mohrin««, in: Engel, Gisela/Kailer, Katja (Hg.), Kolonisierungen und Kolonisationen, Berlin 2004, S. 63-73.

Bugmann, Mirjam/Sarasin, Philipp, »Forel mit Foucault. Rassismus als >Zäsur< im Diskurs von August Forel«, in: Studien und Quellen. Zeitschrift des Schweizerischen Bundesarchivs, Bd. 29: Integration und Ausschluss (2003), S. 43-69.

Casadio, Giovanna, »Fini rivaluta le colonie italiane. Guardate come stanno oggi «, in: La Repubblica vom 26.09.2006, S. 13, (http://ricerca.repubblica.it/repubblica/ archivio/repubblica/2006/09/26/fini-rivaluta-le-colonie-italiane-guardatecome.html), 19.01.2012.

Castro Varela, María do Mar/Dhawan, Nikita, »Mission Impossible. Postkoloniale Theorie im deutschsprachigen Raum«, in: Reuter, Julia/Villa, Paula-Irene (Hg.), Postkoloniale Soziologie. Empirische Befunde, theoretische Anschlüsse, politische Intervention, Bielefeld 2010, S. 303-329.

Dies., Postkoloniale Theorie. Eine kritische Einführung, Bielefeld 2005.

Chakrabarty, Dipesh, Provincializing Europe. Postcolonial Thought and Historical Difference, Princeton 2000.

Christen, Anton, »Libyens Aufständische in der Defensive«, in: Neue Zürcher Zeitung vom 16.03.2011, S. 7 .

Clavin, Patricia, »Defining Transnationalism«, in: Contemporary European History, Jg. 14, H. 4 (2005), S. 421-439.

Comaroff, Jean/Comaroff, John, Ethnography and the Historical Imagination, Boulder 1992.

Comité pour une Nouvelle Histoire de la Suisse (Hg.), Geschichte der Schweiz und der Schweizer, Basel ${ }^{42006 .}$

Comparativ. Zeitschrift für Globalgeschichte und vergleichende Gesellschaftsforschung (Hg.), Entangled Histories. Reflecting on Concepts of Coloniality and Postcoloniality, Jg. 21, H. 1 (2011).

Conrad, Sebastian, Globalisierung und Nation im Deutschen Kaiserreich, München 2006.

Conrad, Sebastian/Eckert, Andreas/Freitag, Ulrike (Hg.), Globalgeschichte. Theorien, Ansätze, Themen, Frankfurt a.M. 2007. 
Conrad, Sebastian/Osterhammel, Jürgen (Hg.), Das Kaiserreich transnational. Deutschland in der Welt, 1871-1914, Göttingen 2004.

Conrad, Sebastian/Randeria, Shalini, »Geteilte Geschichten - Europa in einer postkolonialen Welt«, in: dies. (Hg.), Jenseits des Eurozentrismus, S. 9-49.

Dies. (Hg.), Jenseits des Eurozentrismus. Postkoloniale Perspektiven in den Geschichtsund Kulturwissenschaften, Frankfurt a.M. 2002.

Cooper, Frederick, Colonialism in Question. Theory, Knowledge, History, Berkeley 2005.

David, Thomas/Etemad, Bouda: »Gibt es einen schweizerischen Imperialismus? «, in: Traverse - Zeitschrift für Geschichte, Jg. 5, H. 2 (1998), S. 17-27.

David, Thomas/Etemad, Bouda/Schaufelbuehl, Janick Marina, Schwarze Geschäfte. Die Beteiligung von Schweizern an Sklaverei und Sklavenhandel im 18. und 19. Jahrhundert, Zürich 2005.

Demeulenaere-Douyère, Christiane/Neuschwander, Isabelle et al. (Hg.), Exotiques expositions. Expositions universelles et cultures extra-européennes France 1855-1937, Paris 2010.

Diène, Doudou, »Mission to Switzerland «, in: United Nations General Assembly, http://www.humanrights.ch/home/upload/pdf/070329_Report_Diene_EN.pdf., 30.01.2007.

Dietze, Gabriele, »Critical Whiteness Theory und Kritischer Okzidentalismus. Zwei Figuren hegemonialer Selbstreflexion«, in: dies./Tissberger, Martina/ Hrzán, Daniela/Husmann-Kastein von Lang, Jana (Hg.), Weiß - Weißsein Whiteness. Kritische Studien zu Gender und Rassismus. Critical Studies on Gender and Racism, Frankfurt a.M. 2006, S. 219-247.

Dies., »Postcolonial Theory«, in: Braun, Christina von/Stephan, Inge (Hg.), Gender@Wissen. Eine Handbuch der Gender-Theorien, Böhlau/Köln/Wien 2005, S. 304-324.

Dietze, Gabriele/Brunner, Claudia/Wenzel, Edith (Hg.), Kritik des Okzidentalismus. Transdisziplinäre Beiträge zu (Neo-)Orientalismus und Geschlecht, Bielefeld 2009 .

Dorlin, Elsa, Sexe, race, classe. Pour une épistémologie de la domination, Paris 2009.

Dos Santos Pinto, Jovita/Lienhard, Marina/Purtschert, Patricia: »Die postkoloniale Schweiz. Warum zirkulieren solche Bilder ungestört in einer breiten Öffentlichkeit?«, in: Die Wochenzeitung, Jg. 31, H. 32 (2011), S. 13-15.

Droz, Numa, »Revision der Bundesverfassung Auswanderungs- und Kolonisationswesen, ART. 34«, in: Nationale Kommission für die Veröffentlichung diplomatischer Dokumente der Schweiz (Hg.), Diplomatische Dokumente der Schweiz, Bern 1986, S. 619-621.

Dussel, Enrique, »Beyond Eurocentrism. The World-System and the Limits of Modernity«, in: Jameson, Fredric/Miyoshi, Masao (Hg.), The Cultures of Globalization, Durham 1998, S. 3-31.

Eckert, Andreas, »Der Kolonialismus im europäischen Gedächtnis«, in: Aus Politik und Zeitgeschichte, Jg. 56, H. 1-2 (2008), S. 31-38. 
Eckert, Andreas/Randeria, Shalini, »Geteilte Globalisierung«, in: dies. (Hg.), Vom Imperialismus zum Empire, S. 9-33.

Dies. (Hg.), Vom Imperialismus zum Empire. Nicht-westliche Perspektiven auf Globalisierung, Frankfurt a.M. 2009.

Eckert, Andreas/Wirz, Albert: »Wir nicht, die Anderen auch. Deutschland und der Kolonialismus«, in: Conrad, Sebastian/Randeria, Shalini (Hg.), Jenseits des Eurozentrismus, S. 372-392.

Egli, Martina, »Weder Entschuldigung noch Entschädigung«, in: Die Weltwoche, Jg. 69, H. 23 (2001), S. 16.

Eggers, Maureen Maisha, »Ein Schwarzes Wissensarchiv«, in: dies. et al. (Hg.), Mythen, Masken und Subjekte, S. 18-21.

Eggers, Maureen Maisha/Kilomba, Grada/Piesche, Peggy/Arndt, Susan (Hg.), MYthen, Masken und Subjekte: Kritische Weißseinsforschung in Deutschland, Münster 2005 .

El-Tayeb, Fatima, »Begrenzte Horizonte. Queer Identity in der Festung Europa«, in: Hito Steyerl/Encarnatión Gutiérrez Rodríguez (Hg.), Spricht die Subalterne deutsch?, S. 129-145.

Eriksson Baaz, Maria, The Paternalism of Partnership. A Postcolonial Reading of Identity in Development Aid, London/New York 2005.

Falk, Francesca, Eine gestische Geschichte der Grenze. Wie der Liberalismus an der Grenze an seine Grenzen kommt, Paderborn 2011.

Fanon, Frantz, Schwarze Haut, weiße Masken, Frankfurt a.M. 1980.

Fassin, Eric, De la question sociale à la question raciale? Représenter la société française, Paris 2006.

Fässler, Hans, Reise in Schwarz-Weiss, Schweizer Ortstermine in Sachen Sklaverei, Zürich 2005 .

Feichtinger, Johannes/Prutsch, Ursula/Csaky, Moritz (Hg.), Habsburg postcolonial. Machtstrukturen und kollektives Gedächtnis, Innsbruck/Wien/Bozen 2003.

Feller, Richard/Bonjour, Edgar, Geschichtsschreibung der Schweiz. Vom Spätmittelalter zur Neuzeit, Basel 1979.

Fischer-Tiné, Harald, »Reclaiming savages in >Darkest England < and >Darkest India<. The Salvation Army as Transnational Agent of the Civilizing Mission«, in: Watt, Carey/Mann, Michael (Hg.), Civilizing Missions in Colonial and Postcolonial South Asia, London 2011, S. 125-165.

Ders., »Postkoloniale Studien«, in: Institut für Europäische Geschichte (IEG) (Hg.), Europäische Geschichte Online (EGO), http://www.ieg-ego.eu/de/threads/ europa-und-die-welt/postkoloniale-studien/harald-fischer-tine-postkolonialestudien, 13.01.2010.

Ders., Low and Licentious Europeans. Race, Class and »White Subalternity « in Colonial India, New Delhi 2009.

Franc, Andrea, Wie die Schweiz zur Schokolade kam. Der Kakaohandel der Basler Handelsgesellschaft mit der Kolonie Goldküste (1893-1960), Basel 2008. 
Franzki, Hannah/Aikins, Joshua Kwesi, »Postkoloniale Studien und kritische Sozialwissenschaft«, in: Prokla, Jg. 40, H. 158 (2010), S. 9-28.

Friedrichsmeyer, Sara/Lennox, Sara/Zantop, Susanne (Hg.), The Imperialist Imagination. German Colonialism and its Legacy, Ann Arbor 1998.

Fröhlicher-Stines, Carmel/Mennel, Kelechi Monika, Schwarze Menschen in der Schweiz. Ein Leben zwischen Integration und Diskriminierung. Eine Studie im Auftrag der EKR, Bern 2004.

Gassama, Makhily/Diagne, Mamoussé/Diop, Dialo/Lamko, Koulsy (Hg.), L’Afrique répond à Sarkozy, Paris 2008.

Gerwarth, Robert/Malinowski, Stephan, »Der Holocaust als >kolonialer Genozid<? Europäische Kolonialgewalt und nationalsozialistischer Vernichtungskrieg«, in: Geschichte und Gesellschaft, Jg. 33, H. 3 (2007), S. 439-466.

Groh, Dieter, »Imperialismus«, in: Brunner, Otto/Conze, Werner/Koselleck, Reinhart (Hg.), Geschichtliche Grundbegriffe. Historisches Lexikon zur politisch-sozialen Sprache in Deutschland, Stuttgart 2004, S. 171-221.

Grosse, Pascal, »What does German Colonialism have to do with National Socialism. A Conceptual Framework«, in: Ames, Eric/Klotz, Maria/Wildenthal, Lora (Hg.), Germany's Colonial Past, Lincoln/London 2005, S. 115-134.

Gullestad, Marianne, »Normalising Racial Boundaries. The Norwegian Dispute about the Word Neger«, in: Social Anthropology, Jg. 13, H. 1 (2005), S. 27-46.

Ha, Kien Nghi, »Die kolonialen Muster deutscher Arbeitsmigrationspolitik«, in: Steyerl, Hito/Gutiérrez Rodríguez, Encarnación (Hg.), Spricht die Subalterne deutsch?, S. 56-107.

Ders., »Koloniale Arbeitsmigrationspolitik im Imperial Germany«, in: ders. (Hg.), re/visionen. Postkoloniale Perspektiven von People of Color auf Rassismus, Kulturpolitik und Widerstand in Deutschland, Münster 2007, S. 65-73.

Ha, Kien Nghi/Lauré al-Samarai/Mysorekar, Sheila (Hg.), re/visionen. Postkoloniale Perspektiven von People of Color auf Rassismus, Kulturpolitik und Widerstand in Deutschland, Münster 2007.

Hall, Stuart, »The Spectacle of the >Other «, in: ders. (Hg.), Representation. Cultural Representations and Signifying Practices, London 1997, S. 223-290.

Harries, Patrick, Butterflies \& Barbarians. Swiss Missionaries and Systems of Knowledge in South-East Africa, Oxford 2007.

Ders., »From the Alps to Africa. Swiss Missionaries and Anthropology«, in: Tilley, Helen/Gordon, Robert J. (Hg.), Anthropology, European Imperialism, and the Politics of Knowledge, Manchester 2007, S. 201-224.

Hell, Julia/Steinmetz, George, »The Visual Archive of Colonialism. Germany and Namibia«, in: Public Culture, Jg. 18, H. 1 (2006), S. 141-182.

Hollenstein, Pia, »Interpellation Hollenstein Pia. Schweizer Beteiligung an Sklaverei und transatlantischem Handel mit Sklavinnen und Sklaven«, 03.3014, in: Amtliches Bulletin, Wintersession 2003: http://www.parlament.ch/ab/ frameset/d/n/4701/95679/d_n_4701_95679_95868.htm, 27.04.2011. 
Honold, Alexander/Scherpe, Klaus R., Mit Deutschland um die Welt. Eine Kulturgeschichte des Fremden in der Kolonialzeit, Stuttgart 2004.

Husmann, Jana, Schwarz-Weiß-Symbolik. Dualistische Denktraditionen und die Imagination von »Rasse«, Bielefeld 2010.

Iriye, Akira, »The Internationalization of History«, in: American Historical Review, Jg. 94, H. 1 (1989), S. 1-10.

Iso, Miko, »Weiss - wie Schneewittchen«, in: Olympe. Feministische Arbeitshefte zur Politik, Jg. 15, H. 27 (2008), S. 7-19.

Johnston-Arthur, Araba Evelyn, »>Es ist Zeit, der Geschichte selbst eine Gestalt zu geben $<$. Strategien der Entkolonisierung und Ermächtigung im Kontext der modernen afrikanischen Diaspora in Österreich«, in: Ha, Kien Nghi et al. (Hg.), re/visionen, S. 423-444.

Kalt, Monica, »>Nestlé tötet Babys!< - Tötet Nestlé Babys?«, in: Schaufelbuehl, Janick Marina (Hg.), 1968-1978. Ein bewegtes Jahrzehnt in der Schweiz, Zürich 2009, S. 183-194.

Dies., Tiersmondismus in der Schweiz der 196oer und 1970er Jahre. Von der Barmherzigkeit zur Solidarität, Bern 2010.

Kelertas, Violeta (Hg.), Baltic Postcolonialism, Amsterdam/New York/Rodopi 2006.

Keller, Christoph, »Sieben Schädel und eine Theorie. Die anthropologischen Forschungen Carl Passavants«, in: Schneider, Jürg/Röschenthaler, Ute/Gardi, Bernhard (Hg.), Fotofieber. Bilder aus West- und Zentralafrika. Die Reisen von Carl Passavant 1883-1885, Basel 2005, S. 43-51.

Ders., Der Schädelvermesser. Otto Schlaginhaufen - Anthropologe und Rassenhygieniker. Eine biographische Reportage, Zürich 1995.

Keskinen, Suvi/Tuori, Salla/Irni, Sari/Mulinari, Diana (Hg.), Complying with Colonialism. Gender, Race and Ethnicity in the Nordic Region, Surrey 2009.

Kraft, Claudia/Lüdtke, Alf/Martschukat, Jürgen (Hg.), Kolonialgeschichten. Regionale Perspektiven auf ein globales Phänomen, Frankfurt a.M. 2010.

Kreis, Georg, Die Schweiz und Südafrika 1948-1994. Schlussbericht des im Auftrag des Bundesrates durchgeführten NFP 42+, Bern 2005.

Krüger, Gesine, »Die Weisse und die edlen Wilden. Der Film > Die weisse Massai««, in: Tages-Anzeiger vom 15.09.2005.

Dies., »Vergessene Kriege. Warum gingen deutsche Kolonialkriege nicht in das Historische Gedächtnis der Deutschen ein?«, in: Langewiesche, Dieter/Buschmann, Nikolaus (Hg.), Zur Rolle des Krieges in Gründungsmythen, Frankfurt a.M./New York 2003, S. 120-137.

Kuhn, Konrad, Entwicklungspolitische Solidarität. Die Dritte-Welt-Bewegung in der Schweiz zwischen Kritik und Politik (1975-1992), Zürich 2011.

Kuhn, Konrad/Ziegler, Béatrice, »Die Schweiz und die Sklaverei. Zum Spannungsfeld zwischen Geschichtspolitik und Wissenschaft«, in: Traverse - Zeitschrift für Geschichte, Jg. 16, H. 1 (2009), S. 116-130. 
Kundrus, Birthe, »Kolonialismus. Imperialismus. Nationalsozialismus? Chancen und Grenzen eines neuen Paradigmas«, in: Kraft, Claudia/Lüdtke, Alf/Martschukat, Jürgen (Hg.), Kolonialgeschichten, S. 187-210.

Dies. (Hg.), Phantasiereiche. Zur Kulturgeschichte des deutschen Kolonialismus, Frankfurt a.M. 2003.

Laak, Dirk van, Über alles in der Welt. Deutscher Imperialismus im 19. und 20. Jahrhundert, München 2005 .

Lang, Josef, »Schweizer Initiativen zur Wiedergutmachung der Sklaverei«, o6.3738, Motion vom 19.12.2006, eingereicht im Nationalrat, http://www.parlament. ch/d/suche/seiten/geschaefte.aspx?gesch_id=20063738, 25.01.2012.

Lepenies, Wolfgang, Entangled Histories and Negotiated Universals, Frankfurt a.M. 2003 .

Lindner, Ulrike, »Neuere Kolonialgeschichte und Postcolonial Studies, Version: 1.0«, in: Docupedia-Zeitgeschichte (2011), https://docupedia.de/zg/Neuere_ Kolonialgeschichte_und_Postcolonial_Studies?oldid=77732, 20.01.2011.

Loftsdóttir, Kristín, »Negotiating White Icelandic Identity. Multiculturalism and Colonial Identity Formations«, in: Social Identities, Jg. 17, H. 1 (2011), S. 11-25.

Dies., »The Loss of Innocence. The Icelandic Financial Crisis and Colonial Past«, in: Anthropology Today, Jg. 26, H. 6 (2010), S. 9-13.

Loomba, Ania, Colonialism/Postcolonialism, London 2005.

Lüthi, Barbara, »Coming to Terms with Turns in Rather >Queer Times<. Wozu dienen die Cultural Turns? Review Symposium zu Doris Bachmann-Medicks >Cultural Turns. Neuorientierung in den Kulturwissenschaften««, in: L'Homme. Europäische Zeitschrift für Feministische Geschichtswissenschaft, Jg. 18, H. 2 (2007), S. 131-134.

Lützelschwab, Claude, La Compagnie genevoise des Colonies suisses de Sétif (18531956). Un cas de colonisation privée en Algérie, Bern 2006.

Luxemburg, Rosa, Die Akkumulation des Kapitals. Ein Beitrag zur ökonomischen Erklärung des Imperialismus, Berlin 1913.

Mackenthun, Gesa, »E Pluribus Unum? Die Position der USA im postkolonialen Diskurs«, in: Das Argument - Zeitschrift für Philosophie und Sozialwissenschaften, Jg. 38, H. 215 (1996), S. 373-379.

Maissen, Thomas, Geschichte der Schweiz, Baden 2010.

Maß, Sandra, Weiße Helden, schwarze Krieger. Zur Geschichte kolonialer Männlichkeit in Deutschland 1918-1964, Köln/Weimar 2006.

Matare, Eleonora/Schneider, Jürg/Zeugin, Bettina (Hg.), Black, Noir, Schwarz. Zwölf Porträts aus Basel, Basel 2002.

Mattioli, Aram, »Viva Mussolini!«. Die Aufwertung des Faschismus im Italien Berlusconis, Zürich 2010.

Mbembe, Achille, »Provincializing France?«, in: Public Culture, Jg. 23, H. 1 (2011), S. 85-119.

Ders., »L'afrique de Nicolas Sarkozy«, in: africultures, 01.08.2007, http://www. africultures.com/php/index.php?nav=article\&no=6784, o8.11.2010. 
McClintock, Anne, Imperial Leather. Race, Gender and Sexuality in the Colonial Contest, New York/London 1995.

Meier-Mesquita, Cintia, »Die soziale Bedeutung der äusseren Erscheinung. >Farbige $<$ in einer >nichtfarbigen< Gesellschaft«, in: Tangram. Bulletin der Eidgenössischen Kommission gegen Rassismus, Jg. 5, H. 8 (2000), S. 7-10.

Menrath, Manuel, Exotische Soldaten und ehrbare Töchter. Triengen 1940 - afrikanische Spahis in der Schweiz, Zürich 2010.

Mergel, Thomas, »Modernisierung«, in: Institut für Europäische Geschichte (IEG) (Hg.), Europäische Geschichte Online (EGO), http://www.ieg-ego.eu/mergelt2011-de, 27.04.2011.

Mesquita, Sushila: Ban Marriage! Ambivalenzen der Normalisierung aus queer-feministischer Perspektive, Wien 2011.

Mezzadra, Sandro: »Bringing the Capital Back in. A Materialist Turn in Postcolonial Studies?«, in: Inter-Asia Cultural Studies, Jg. 12, H. 1 (2011), S. 154-164.

Ders., La condizione postcoloniale. Storia e politica nel presente globale, Verona 2008.

Michel, Noémi/Honegger, Manuela, »Thinking Whiteness in French and Swiss Cyberspaces«, in: Social Politics, Jg. 17, H. 4 (2010), S. 423-449.

Minder, Patrick, La Suisse coloniale? Les représentations de l'Afrique et des Africains en Suisse au temps des colonies (1880-1939), Bern 2009.

Ders., »La construction du colonisé dans une métropole sans empire. Le cas de la Suisse (1880-1939)«, in: Bancel, Nicolas/Blanchard, Pascal/Boëtsch, Gilles/ Deroo, Eric (Hg.), Zoos humains. Au temps des exhibitions humaines, Paris 2004, S. 227-234.

Mitchell, Timothy, »Die Welt als Ausstellung«, in: Conrad, Sebastian/Randeria, Shalini (Hg.), Jenseits des Eurozentrismus, S. 148-176.

Mohanty, Chandra, Feminism without Borders, Durham/London 2003.

Mollenhauer, Daniel, »Erinnerungspolitik in der postkolonialen Republik - Frankreich und das koloniale Erbe«, in: Kraft, Claudia/Lüdtke, Alf/Martschukat, Jürgen (Hg.), Kolonialgeschichten, S. 117-141.

N. N., »Weisse Waren«, in: Der Globi. Jugendschrift der Magazine zum Globus, Jg. 1, H. 2 (1935), o.S.

Ochsenbein, Ulrich, »Präsidialvortrag von Ulrich Ochsenbein bei Eröffnung der Tagsatzung«, in: Bonjour, Edgar (Hg.), Die Gründung des schweizerischen Bundesstaates, Basel 1948, S. 213-220.

Olympe. Feministische Arbeitshefte zur Politik (Hg.), Wider die Instrumentalisierung von Frauenrechten. Burkaverbot: Feministische Positionen und Analysen, Jg. 17, H. 31 (2010).

Osterhammel, Jürgen, Kolonialismus. Geschichte - Formen - Folgen, München 2009.

Ders., »Imperialismus«, in: Gosepath, Stefan/Hinsch, Wilfried/Rössler, Beate (Hg.), Handbuch der Politischen Philosophie und Sozialphilosophie, Berlin 2008, S. $534-538$.

Ders., »Imperialgeschichte«, in: Cornelißen, Christoph (Hg.), Geschichtswissenschaften. Eine Einführung, Frankfurt a.M. 2000, S. 221-232. 
Palmberg, Mai, »The Nordic Colonial Mind«, in: Keskinen, Suvi et al., Complying with Colonialism, S. 35-50.

Patel, Kiran Klaus, »Transnationale Geschichte - ein neues Paradigma«, in: geschichte.transnational, 02.02.2005, http://geschichte-transnational.clio-online. net/forum/type=diskussionen\&id=573, 25.01.2012.

Perrenoud, Marc, »Aperçu sur les Suisses de l'étranger et la décolonisation en Afrique«, in: Studien und Quellen. Zeitschrift des Schweizerischen Bundesarchivs, Bd. 28 (2002): Die Auslandschweizer im 20. Jahrhundert, S. 327-344.

Ders., »Algerien«, in: Jorio, Marco (Hg.), Historisches Lexikon der Schweiz, Basel 2001, S. 182-183.

Poddar, Prem/Patke, Rajeev S./Jensen, Lars (Hg.), Postcolonial Literatures. Continental Europe and its Empires, London 2011.

Porter, Andrew, European Imperialism, 1860-1914, Basingstoke 1994.

Pous, Jacques, Henry Dunant, l'Algérien ou le mirage colonial, Genf 1979.

Priester, Karin, Rassismus. Eine Sozialgeschichte, Leipzig 2003.

Puar, Jasbir K., Terrorist Assemblages: Homonationalism in Queer Times, Durham 2007.

Purtschert, Patricia: »Chewing on Post_colonial Switzerland. Redigesting what has not yet been swallowed, Part III«, in: Thal, Andrea (Hg.): Chewing the Scenery 3rd Edition, Zürich 2011, S. 41-44, 67-70.

Dies.: »Chewing on Post_colonial Switzerland. Redigesting what has not yet been swallowed, Part II«, in: Thal, Andrea (Hg.): Chewing the Scenery and Edition, Zürich 2011, S. 95-100, 121-126, 149-154.

Dies.: »Chewing on Post_colonial Switzerland. Redigesting what has not yet been swallowed, Part I«, in: Thal, Andrea (Hg.): Chewing the Scenery 1st Edition, Zürich 2011, S. 173-176, 199-202.

Dies., »>Heute bedankt sich Naresh Khan bei Silvia Hug für ihr Engagement<. Notizen zur postkolonialen Schweiz«, in: Olympe. Feministische Arbeitshefte zur Politik, Jg. 15, H. 27 (2008), S. $76-87$.

Dies., »Postkoloniale Diskurse in der Schweiz. >De Schorsch Gaggo reist uf Afrika««, in: Widerspruch, Jg. 28, H. 54 (2008), S. 169-180.

Reinhardt, Volker, Geschichte der Schweiz, München ${ }^{4} 2010$.

Richter, Julius, »Die Bedeutung der Mission für unsere Kolonien«, in: Evangelisches Missions-Magazin, Bd. 83 (1898), S. 309-323.

Romeo, Caterina, »Postcolonial Italy. The Colonial Past in Contemporary Italy«, in: Dept. of English, University of Pennsylvania, http://call-for-papers.sas.upenn. edu/node/34944, o6.02.2011.

Rossfeld, Roman, Schweizer Schokolade. Industrielle Produktion und kulturelle Konstruktion eines nationalen Symbols 1860-1920, Baden 2007.

Ruffieux, Roland: »Die Schweiz des Freisinns (1848-1914)«, in: Mesmer, Beatrix/ Favez, Jean-Claude/Broggini, Romano (Hg.): Geschichte der Schweiz und der Schweizer, Basel 1986, S. 639-730.

Said, Edward, Culture and Imperialism, London 1993. 
Ders., Orientalism. Western Conceptions of the Orient, London 1978.

Salgado, Rubia: »Chewing the Borders. Oder Kauen, um wach zu bleiben. Oder Widerstand im Widerspruch «, in: Thal, Andrea (Hg.): Chewing the Scenery and Edition, Zürich 2011, S. 199-206.

Schär, Bernhard C., »Karies, Kulturpessimismus und KVG. Zur Geschichte der Zahnmedizin in der Schweiz«, in: Traverse - Zeitschrift für Geschichte, Jg. 15, H. 2 (2008), S. 99-116.

Ders., »Mariella Mehr als Inspiration für eine postkoloniale Geschichte der Schweiz - einige Überlegungen«, in: Ruchat, Anna (Hg.), Lieblebchen sag - 60 Jahre Mariella Mehr, Riva San Vitale 2007, S. 8-16.

Schneider, Jürg/Lüthi, Barbara, »Carl Passavant (1854-1887). Eine Welt in Bildern«, in: Traverse - Zeitschrift für Geschichte, Jg. 14, H. 3 (2007), S. 113-122.

Schneider, Jürg/Röschenthaler, Ute/Gardi, Bernhard (Hg.), Fotofieber. Bilder aus West- und Zentralafrika. Die Reisen von Carl Passavant 1883-1885, Basel 2005.

Schroer, Markus, »Gewalt ohne Gesicht. Zur Notwendigkeit einer umfassenden Gewaltanalyse«, in: Heitmeyer, Wilhelm/Soeffner, Hans-Georg (Hg.), Gewalt. Entwicklungen, Strukturen, Analyseprobleme, Frankfurt a.M. 2004, S. 151-173.

Schuhmann, Antje, »Exoticizing the Erotic. White on White via the Black Body: Collecting Artefacts within German Dominant Culture«, in: Wright, Michelle/ Schuhmann, Antje (Hg.), Blackness and Sexualities, Berlin 2007, S. 9-125.

Schwarz, Henry/Ray, Sangeeta (Hg.), A Companion to Postcolonial Studies, Malden 2000.

Sciolino, Elaine, »Immigration, Black Sheep and Swiss Rage«, in: New York Times, 08.10.2007, http://www.nytimes.com/2007/10/08/world/europe/o8swiss.html, 25.01.2012.

Skenderovic, Damir/D’Amato, Gianni, Mit dem Fremden politisieren. Rechtspopulistische Parteien und Migrationspolitik in der Schweiz seit den $1960 e r$ Jahren, Zürich 2008.

Spivak, Gayatri, Can the Subaltern Speak? Postkolonialität und subalterne Artikulation, Wien 2007.

Dies., In Other Worlds. Essays in Cultural Politics, New York/London 1988.

Staehelin, Balthasar, Völkerschauen im Zoologischen Garten Basel 1879-1935, Basel 1994 .

Steiner, Yvonne, Henry Dunant. Biographie, Herisau 2010.

Steinmetz, George, »Decolonizing German Theory. An Introduction«, in: Postcolonial Studies, Jg. 9, H. 1 (2006), S. 3-13.

Stettler, Niklaus/Haenger, Peter/Labhardt, Robert, Baumwolle, Sklaverei und Kredite. Die Basler Welthandelsfirma Burckhardt a Cie. in revolutionärer Zeit 1789-1815, Basel 2004.

Steyerl, Hito/Gutiérrez Rodríguez, Encarnación, »Einleitung«, in: dies. (Hg.), Spricht die Subalterne deutsch?, S. 7-16.

Dies. (Hg.), Spricht die Subalterne deutsch? Migration und postkoloniale Kritik, Münster 2003 . 
Stoler, Ann Laura/Cooper, Frederick, »Between Metropole and Colony. Rethinking a Research Agenda«, in: dies. (Hg.), Tensions of Empire. Colonial Cultures in a Bourgeois World, Berkeley 1997, S. 1-56.

Stucki, Lorenz, Das heimliche Imperium. Wie die Schweiz reich wurde, Bern 1968.

Tanner, Jakob, »Tunnelblick«, in: Kwaschik, Anne/Wimmer, Mario (Hg.), Von der Arbeit des Historikers. Ein Wörterbuch zu Theorie und Praxis der Geschichtswissenschaft, Bielefeld 2010, S. 203-208.

Thürmer-Rohr, Christina, Vagabundinnen - Feministische Essays, Berlin 1987.

Trinh, Minh-ha, Woman, Native, Other, Bloomington 1989.

Triulzi, Alessandro: »Dispacing the Colonial Event. Hybrid Memories of Postcolonial Italy«, in: De Donno, Fabrizio/Srivastava, Neelam (Hg.), Colonial and Postcolonial Italy. Special Issue of Interventions, Jg. 8, H. 3 (2006), S. 430-443.

Tyrell, Ian, Transnational Nation. United States History in Global Perspective Since 1789 , Basingstoke 2007.

Vallely, Paul, »Switzerland. Europe's Heart of Darkness?«, in: The Independent vom 07.09.2007, http://www.independent.co.uk/news/world/europe/switzerlandeuropes-heart-of-darkness-401619.html, 25.04.2011.

Vögelin, Salomon, »Rede zur Beleuchtung der Motion Zemp und Genossen auf Revision der Bundesverfassung, gehalten im Nationalrathe den 21. Juni 1884«, in: Isler, Alexander (Hg.), Prof. Dr. Salomon Vögelin, Nationalrath. Lebensbild eines schweizerischen Volksmannes, Winterthur 1892, S. 43-61.

Vuorela, Ulla, »Colonial Complicity. The >Postcolonial in a Nordic Context«, in: Keskinen, Suvi et al., Complying with Colonialism, S. 19-33.

Walgenbach, Katharina, Die weiße Frau als Trägerin deutscher Kultur. Koloniale Diskurse über Geschlecht, >Rasse< und Klasse im Kaiserreich, Frankfurt a.M. 2005.

Wälti, Simon, »Agassizhorn wird nicht Rentyhorn umgetauft«, in: Der Bund vom 23.08.2010, http://www.derbund.ch/bern/Agassizhorn-wird-nicht-inRentyhorn-umgetauft-/story/20357794, 25.01.2012.

Weinstein, Barbara, »History Without a Cause? Grand Narratives, World History, and the Postcolonial Dilemma«, in: International Review of Social History, Jg. 50, H. 1 (2005), S. 71-93.

Wendland, Anna Veronika, »Imperiale, koloniale und postkoloniale Blicke auf die Peripherien des Habsburgerreiches«, in: Kraft, Claudia/Lüdtke, Alf/Martschukat, Jürgen (Hg.), Kolonialgeschichten, S. 211-235.

Wirz, Albert, »Die Humanitäre Schweiz im Spannungsfeld zwischen Philantrophie und Kolonialismus. Gustave Moynier, Afrika und das IKRK«, in: Traverse Zeitschrift für Geschichte, Jg. 5, H. 2 (1998), S. 95-110.

Witschi, Beat, Schweizer aufimperialistischen Pfaden. Die schweizerischen Handelsbeziehungen mit der Levante 1848 bis 1914, Stuttgart 1987.

Wolfe, Patrick, »History and Imperialism. A Century of Theory. From Marxism to Postcolonialism«, in: American Historical Review, Jg. 102, H. 2 (1997), S. 388420. 
Young, Robert J. C., Postcolonialism. An Historical Introduction, Oxford 2001.

Zangger, Andreas, Koloniale Schweiz. Ein Stück Globalgeschichte zwischen Europa und Südostasien (1860-1930), Bielefeld 2011.

Ziegler, Béatrice, Schweizer statt Sklaven. Schweizerische Auswanderer in den KaffeePlantagen von São Paulo (1852-1866), Stuttgart 1985.

Ziegler, Jean, Das Schweizer Imperium. Banken und Banditen, Fluchtgeld-Skandal, Profite aus Hunger und Krieg, Kolonialismus im eigenen Land, Reinbek b. Hamburg 1982.

Zimmerer, Jürgen, »Die Geburt des >Ostlandes< aus dem Geist des Kolonialismus. Ein postkolonialer Blick auf die NS-Eroberungs- und Vernichtungspolitik «, in: Sozial.Geschichte. Zeitschrift für die historische Analyse des 20. und 21. Jahrhunderts, Jg. 2, H. 1 (2004), S. 10-43.

Zimmerer, Jürgen/Zeller, Joachim (Hg.), Völkermord in Deutsch-Südwestafrika. Der Kolonialkrieg (1904-1908) in Namibia und seine Folgen, Berlin 2003.

\section{AbbildungsnachWeise}

Abb. 1 Fotografie der Werbung für »Weiße Waren«, Hauszeitung Globus, Juni 1933, S. 226

Abb. 2 SVP-Plakat für die Ausschaffungsinitiative 2007

Abb. 3 Moutons de Garde 2007, http://www.moutonsdegarde.ch/mdg/view. php, 25.04.2011

Abb. 4 Das schwarze Schaf 2007, http://www.das-schwarze-schaf.ch, 25.04.2011 
